\title{
Multiomics Characterization of Potential Therapeutic Vulnerabilities in Low-grade Serous Ovarian Carcinoma
}

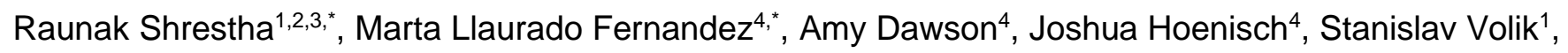
Yen-Yi Lin ${ }^{1,2}$, Shawn Anderson ${ }^{1}$, Hannah Kim², Anne Haegert ${ }^{1}$, Shane Colborne ${ }^{5}$, Brian McConeghy ${ }^{1}$, Robert H. Bell ${ }^{1}$, Sonal Brahmbhatt ${ }^{1}$, Gabriel E. DiMattia ${ }^{6}$, Stephane Le Bihan ${ }^{1}$, Gregg B. Morin ${ }^{5,7}$, Colin C. Collins ${ }^{1,2, \dagger}$, and Mark S. Carey ${ }^{4, \dagger}$

${ }^{1}$ Vancouver Prostate Centre, 2660 Oak St, Vancouver, BC V6H 3Z6, Canada.

${ }^{2}$ Department of Urologic Sciences, University of British Columbia, 2775 Laurel Street, Vancouver, BC V5Z 1M9, Canada.

${ }^{3}$ Department of Radiation Oncology, University of California San Francisco, 1825 4th St., San Francisco, CA 94158, USA.

${ }^{4}$ OvCaRe group, Department of Gynecologic Oncology, University of British Columbia, 1125 Howe Street, Vancouver, BC V6Z 2K8, Canada.

${ }^{5}$ Michael Smith Genome Sciences Centre, BC Cancer Agency, 570 W 7th Ave, Vancouver, BC V5Z 4 S6 Canada.

${ }^{6}$ Translational Ovarian Cancer Research Program, London Health Science Centre, 339 Windermere Road, London, ON N6A 5A5, Canada.

${ }^{7}$ Department of Medical Genetics, University of British Columbia, 4500 Oak St, Vancouver, BC V6H 3N1, Canada.

*These authors are co-first authors and contributed equally to this work.

†Corresponding and joint senior authors ccollins@prostatecentre.com; mark.carey@ubc.ca

Running Title: Multiomics Characterization of Low-grade Serous Ovarian Cancer 


\section{Abstract}

Background: Low-grade serous ovarian carcinoma (LGSOC) is a rare tumor subtype with high case fatality rates. As such, there is a pressing need to develop more effective treatments using newly available preclinical models for therapeutic discovery and drug evaluation. Here, we use a multiomics approach to interrogate a collection of LGSOC patient-derived cell lines to elucidate novel biomarkers and therapeutic vulnerabilities.

Methods: Fourteen LGSOC cell lines were interrogated using whole exome sequencing, RNA sequencing, and mass spectrometry-based proteomics. Somatic mutation, copy-number aberrations, gene and protein expression were analyzed and integrated using different computational approaches. LGSOC cell line data was compared to publicly available LGSOC tumor data (AACR GENIE cohort), and also used for predictive biomarker identification of MEK inhibitor (MEKi) efficacy. Protein interaction databases were evaluated to identify novel therapeutic targets.

Results: KRAS mutations were exclusively found in MEKi-sensitive and NRAS mutations mostly in MEKiresistant cell lines. Analysis of COSMIC mutational signatures revealed distinct patterns of nucleotide substitution mutations in MEKi-sensitive and MEKi-resistant cell lines. Deletions of $C D K N 2 A / B$ and MTAP genes (chromosome 9p21) were much more frequent in cell lines than tumor samples and possibly represent key driver events in the absence of KRAS/NRAS/BRAF mutations. For in-vitro MEKi efficacy prediction, proteomic data provided better discrimination than gene expression data. Condensin, MCM, and RFC protein complexes were identified as potential treatment targets in MEKi-resistant cell lines.

Conclusions: Our LGSOC cell lines are representative models of the most common molecular aberrations found in LGSOC tumors. This study highlights the importance of using proteomic data in multiomics assessment of drug prediction and identification of potential therapeutic targets. CDKN2A/B and MTAP deficiency offer an opportunity to find synthetically lethal candidates for novel treatments. Multiomics approaches are crucial to improving our understanding of the molecular aberrations in LGSOC, establishing effective drug prediction programs and identifying novel therapeutic targets in LGSOC.

Keywords: Low-grade Serous Ovarian Cancer, MAPK pathway, MEK inhibitor, and Drug resistance 


\section{Background}

Low-grade serous ovarian carcinoma (LGSOC) is a rare subtype of epithelial ovarian cancer with a poor prognosis. Women with LGSOC are usually diagnosed with advanced-stage disease and only 10-20\% are alive 10 years after diagnosis [1,2]. Research on LGSOC is challenging due to its low prevalence, uncertain etiology, and the limited availability of research models. However, in the last 5-10 years investigators have elucidated many key genomic aberrations, leading to major advancements in the molecular characterization and classification of LGSOC [3,4]. In contrast to other ovarian cancer subtypes, LGSOC are genetically characterized by high frequency of oncogenic mutations in KRAS, NRAS, and BRAF (20-40\%, 7-26\%, 5-33\%, respectively) [5-7], a very low prevalence of TP53 mutations $(<8 \%$ [8,9], frequent copy-number deletion of CDKN2A resulting in loss of tumor suppressor protein $\mathrm{p} 16$ $(15-53 \%)[10,11]$, and high expression of estrogen receptor (ER) and progesterone receptor (PR) (>90\% and $>50 \%$, respectively) [12-14]. More recently, less frequent mutations in USP9X (15\%) and EIF1AX $(8 \%)$ have been described in a small proportion of LGSOC tumors $[10,15]$.

Traditionally, the therapeutic management of different ovarian cancer histotypes has been similar as it is now appreciated that different ovarian histological subtypes represent different diseases [16]. Consequently, the most common treatment for LGSOC is based on cytoreductive surgery followed by platinum/paclitaxel chemotherapy. However, unlike the most common subtype of epithelial ovarian cancer (high-grade serous ovarian carcinoma or HGSOC), LGSOC is relatively resistant to standard chemotherapy [2,17]. Anti-estrogen therapy is also a common therapy though response rates for the treatment of recurrent disease patients are low (9-14\%) [18]. In this context, the identification of oncogenic mutations affecting MAPK genes (RAS/RAF/MEK) led to the evaluation of targeted agents such as MEK inhibitors (MEKi) in clinical trials of patients with LGSOC. Recently, a study using the MEKi trametinib for the treatment of relapsed LGSOC, demonstrated a statistically significant improvement in progression free survival (PFS) when compared to standard of care therapies. Trametinib monotherapy resulted in an improvement in PFS of almost 6 months (13 vs 7.2 months) with objective response rates of $26 \%$ (compared to $6 \%$ in the standard treatment arm). While promising, these results demonstrated that benefit of trametinib therapy is restricted to a subgroup of patients. Thus, additional effective therapies are needed.

Advancements in the treatment of rare cancers such as LGSOC rests on a better understanding of their unique molecular features and how they can be exploited for therapeutic gain. Unfortunately, results from genomic precision oncology clinical trials have shown that only $5-7 \%$ of cancer patients derive benefit 
$[19,20]$. However, in this small fraction of patients, response rates were over $50 \%$ and durable response durations of 30 months were observed. To accelerate progress in precision oncology, future research must go beyond the genomic aberrations found in tumors and explore the more complex aspects of tumor biology. Thus, multiomics tumor profiling with data integration analysis [21] provides a new window of opportunity for the identification of molecular disease drivers leading to more successful drug therapies. This approach can be extremely useful in the study of rare cancers such as LGSOC, where there is an urgent need for more effective therapies.

In the last few years, we have established a large collection of cancer cell line models from advanced/recurrent LGSOC patients [22]. These models have since then been used for pre-clinical drug testing and biomarker discovery in several studies [22-24]. With the introduction of MEKi therapy for LGSOC, there is a pressing need to identify biomarkers that predict tumor response. These predictive biomarkers will not only allow us to identify those patients that are more likely to benefit from MEKi therapy, and through further research identify novel treatment targets for the resistant patient population [24].

In this study, we performed an integrative analysis of the genomes, transcriptomes, and proteomes of 11 clinically characterized LGSOC cell line models with the goal to identify novel tumor vulnerabilities for the development of future drug treatments. Here, using multiomics approach, we aim to: (1) characterize the molecular complexity of our LGSOC models, (2) elucidate novel potential drivers associated with LGSOC disease, and (3) identify molecular biomarkers that predict response to MEKi therapy. 


\section{Methods}

\section{Tumor samples and clinical information}

Advanced or recurrent LGSOC samples (tumor and ascites) were obtained from the OvCaRe gynecologic tumor bank (Vancouver General Hospital (VGH), British Columbia Cancer Agency, and the John and Mary Knight Translational Ovarian Cancer Research Unit (London Regional Cancer Program, London, Ontario, Canada). Tumor bank protocols, cell line derivation, and all research relating to this study was approved by institutional human ethics review boards at BCCA (H14-02859), the University of British Columbia (UBC; R05-0119), and the University of Western Ontario (HSREB 12668E). Clinical information was extracted retrospectively from patient records. Tumor histology was confirmed by a gynecological pathologist.

\section{Patient-derived LGSOC cell lines}

Low-grade serous ovarian cancer patient-derived cell lines were established through continuous in-vitro culture of patient material obtained through OvCaRe or the John and Mary Knight Translational Ovarian Cancer Research Unit (cell line iOvCa241) tumor banks [22,24]. For details, see Supplementary Methods.

\section{Whole exome sequencing}

DNA was extracted from LGSOC cell lines using an AllPrep DNA/RNA Mini Kit (Qiagen, Toronto, ON), and from matched patient buffy coat samples using a DNeasy Blood and Tissue Kit (Qiagen, Toronto, $\mathrm{ON}$ ), according to the manufacturer's protocol. DNA concentration was quantified using a Qubit ${ }^{\circledR} \mathrm{dsDNA}$ HS Assay (Thermo Scientific, Burlington, ON, Canada). About $0.5 \mu \mathrm{g}$ of genomic DNA was fragmented by hydrodynamic shearing (Covaris, Inc., Woburn, MA, USA) to generate 150-280 bp fragments. After end repair, fragments were ligated to Illumina barcoded adapters, cleanup, amplified by polymerase chain reaction (PCR) to enrich for adapter ligated fragments, and controlled for quality. The library was then enriched by liquid phase hybridization using Agilent SureSelect XT Human All Exon v6 (Agilent Technologies, CA, USA) following the manufacturer's recommendations. The captured library was amplified by PCR using indexing primers, cleanup and quality assessment was done with the TapeStation 4200 (Agilent). Libraries are submitted to PE100 sequencing on Illumina HiSeq4000.

\section{Somatic variant calling}


Sequence alignment and mutation calling were performed in Partek Flow environment (@ Partek Inc). Sequence reads were aligned to the GRCh38/hg38 human genome build using bwa 0.7.2 [25]. Variants were called using Strelka 1.0.15 [26] for all cell lines except for CL-02 (lacking buffy coat sample). CL-02 variant calling was performed using LoFreq 2.1.3.a [27]. The called variants were annotated using the Annovar software [28]. Annotated calls were then filtered to show only protein-changing single nucleotide variations (SNVs) that were present in cell line DNA at allele frequencies (AF) greater than 0.1 and coverage higher than 16x. For CL-02, all calls not present in dbSNP (version 138) were retained, while of the calls that were present in dbSNP, calls with (average heterozygosity + aveHet standarderror) less than 0.1 were retained. These were additionally filtered using the same criteria as for the Strelka calls.

\section{Copy number aberration (CNA) calls}

Data analysis was performed using Nexus Copy Number Discovery Edition Version 9.0 (BioDiscovery, Inc., El Segundo, CA). Samples were processed using the Nexus NGS functionality with the FASST2 segmentation. The log ratio thresholds for single copy gain and single copy loss were set at +0.18 and 0.18 , respectively. The log ratio thresholds for gain of 2 or more copies and for a homozygous loss were set at +0.6 and -1.0 , respectively. Tumor sample Binary alignment map (BAM) files were processed with corresponding normal tissue BAM files. Probes were normalized to the median.

\section{Whole transcriptome sequencing (RNA-seq)}

Total RNA from was isolated in accordance with the mirVana miRNA Isolation kit (ThermoFisher). Strand specific RNA sequencing was performed on quality controlled high RIN value (>7) RNA samples (Bioanalyzer Agilent Technologies). In brief, 200ng of total RNA was first treated to remove the rRNA and then purified using the Agencourt RNA Clean XP Kit (Beckman Coulter) prior to analysis with the Agilent RNA 6000 Pico Chip to confirm rRNA removal. Next, the rRNA-depleted RNA was fragmented and converted to cDNA. Subsequent steps include end repair, addition of an 'A' overhang at the 3' end, ligation of the indexing-specific adaptor, followed by purification with Agencourt Ampure XP beads. The strand specific RNA library prepared using TruSeq stranded mRNA kit (Illumina). Libraries were quality checked and sized with a TapeStation 4200 (Agilent Technologies), then quantified using the KAPA Library Quantification Kit for Illumina platforms (Kapa Biosystems). Libraries were submitted to PE75 sequencing on Illumina NextSeq500. About 20-50 million sequencing reads per library was analyzed for each sample.

\section{Transcriptome (RNA-seq) quantification}


RNA-seq sequence alignment and transcript/gene quantification were performed in Partek Flow environment (C Partek Inc). The RNA-seq reads were first trimmed to remove low quality bases from the 3' end. The trimmed reads were then mapped to the GRCh38/hg38 human reference genome and transcriptome (RefSeq release 83) using splice-aware aligner STAR (2.5.3a) [29]. Based on the reads that can only be mapped to a single genomic location, the transcript/gene expression quantification was performed using HTSeq-count [30]. Cross-sample normalization of expression values were done by DESeq [31].

\section{Proteomics analysis using mass spectrometry}

Seven LGSOC cell lines were selected for global proteome analysis (Supplementary Table 1) in triplicates using 3 separately cultured samples for each. Cultures of 5 different HGSOC cell lines (OVCAR8, OVSAYO, CAOV3, OVCAR4, and VOA2900) were also used as a control. VOA2900 is a HGSOC cell line developed by the ovarian cancer research program at VGH. Cells were collected by trypsinization and washed with $1 \mathrm{X}$ dPBS. A minimum of $1.0 \times 10^{6}$ cells per cell line were used in these analyses. Cells pellets were lysed in $200 \mathrm{uL}$ of a buffer containing guanidine hydrochloride (4M), HEPES $(\mathrm{pH}$ 8.5, $50 \mathrm{mM})$, 2-chloroacetamide $(40 \mathrm{mM})$, tris(2-carboxyethyl) phosphine (10 mM), ethylenediaminetetraacetic acid $(5 \mathrm{mM})$, 1x phosphatase inhibitor, and 1x protease inhibitor. Bead beating of the cells was performed in Lysing Matrix D tubes on a FastPrep24 (6 M/s for $45 \mathrm{~s}, 60 \mathrm{~s}$ rest, 6 $\mathrm{M} / \mathrm{s}$ for $45 \mathrm{~s}$ ). Cell lysate was then heated at $95 \mathrm{C}$ for $15 \mathrm{~min}$ with $1200 \mathrm{rpm}$ mixing. $50 \mathrm{ug}$ of each sample was diluted 10x in 50 mM HEPES pH 8.5 (concentration determined using by BCA assay) and digested using a 50:1 ratio of protein:trypsin/Lys-C mixture at $37 \mathrm{C}$ overnight with shaking at $1200 \mathrm{rpm}$. A small aliquot of each LGSOC sample was mixed and analyzed as LGSOC-mixed control. The five HGSOC samples were mixed and used as a HGSOC-mixed control sample. An aliquot of all the LGSOC and HGSOC samples were mixed and used as an all sample pooled internal standard. Tandem mass tag (TMT10) labeling was performed by adding 100 ug TMT label in acetonitrile and incubating at room temperature for thirty minutes twice. Excess label was quenched with $10 \mathrm{uL}$ of $1 \mathrm{M}$ glycine. Individual TMT channels were combined and the volume of the combined TMT labeled sample was reduced to 10$20 \%$ of the original volume. Three 10-plex batches were prepared, where each batch contained one replicate of each of the LGSOC samples, the LGSOC-mixed control, the HGSOC mixed control, and the all sample pooled internal standard. The combined samples were fractionated by high $\mathrm{pH}$ reverse-phase high performance liquid chromatography (HPLC) in a gradient of acetonitrile and $20 \mathrm{mM}$ ammonium bicarbonate in water into 48 individual fractions. These 48 fractions were concatenated into 12 fractions by combining every twelfth fraction, each of which was analyzed by low $\mathrm{pH}$ nanoLC mass spectrometry (MS) using a MS/MS/MS $\left(\mathrm{MS}^{3}\right)$ method on a Thermo Scientific Easy $\mathrm{nLC}$ coupled to a ThermoFisher 
Orbitrap Fusion MS. The Uniprot Human Proteome was used as a reference proteome [32]. Only peptides that were identified in all three batches were retained. ComBat, a function from sva R-package [33], was used to adjust for batch effects using an empirical Bayes adjustment. Detection of differentially expressed proteins from the collected peptide data was determined by the Probe-level Expression Change Averaging (PECA) in R [34].

\section{Mutational signature analysis}

We used deconstructSigs [35], a multiple regression approach to statistically quantify the contribution of mutational signature for each tumor. The 30 mutational signatures were obtained from the COSMIC mutational signature database [36]. In brief, deconstructSigs attempts to recreate the mutational pattern using the trinucleotide mutation context from the input sample that closely resembles each of the 30 mutational signatures from COSMIC mutational signature database (v2 - March 2015). In this process, each mutational signature is assigned a weight normalize between 0 to 1 indicating its contribution. Only those mutational signatures with a weight more than 0.06 were considered for analysis. We further combined (or grouped) these 30 mutational signatures based on the similarity of their mutational features or functional associations. For details, see Supplementary Methods.

\section{Prioritization of driver genes using HIT'nDRIVE}

HIT'nDRIVE [37] measures the potential impact of genomic aberrations on changes in the global expression of other genes/proteins which are in close proximity in a gene/protein-interaction network. It then prioritizes those aberrations with the highest impact as cancer driver genes. Both non-silent somatic mutation calls and CNA gain or loss were independently collapsed in gene-patient alteration matrix with binary labels. mRNA and protein expression values were used to derive expression-outlier gene-patient outlier matrix using GESD test. STRING ver10 [38] protein-interaction network was used to compute pairwise influence value between the nodes in the interaction network. We integrated these genome and transcriptome as well as genome and proteome data using HIT'nDRIVE. First we integrated genome and transcriptome data. Here we ran HIT'nDRIVE separately using SNV-mRNA expression data and CNAmRNA expression data. For this we used the parameters: $\boldsymbol{\alpha}=0.9, \boldsymbol{\beta}=0.7$, and $\boldsymbol{\gamma}=0.7$. Next, we integrated genome and proteome data. Here we ran HIT'nDRIVE separately using SNV-protein expression data and CNA-protein expression data. For this we used the parameters: $\boldsymbol{\alpha}=0.9, \boldsymbol{\beta}=0.7$, and $\boldsymbol{\gamma}=0.8$. We used IBM-CPLEX as the Integer Linear Programming solver. The results were later combined to downstream analysis. 


\section{Differential expression analysis}

Differential expression analysis of MEKi-response phenotypes was performed by applying linear empirical Bayes model using "sva" R-package [33]. Gene (mRNA) and protein expression data of the MEKi-response phenotypes were used for this purpose. We used the following threshold values to select genes and proteins for MEKi response analysis and downstream pathway analysis: for mRNA expression data, pvalue $\leq 0.05$ and $\mid$ foldchange $\mid>1.03$, and for protein expression data, pvalue $\leq 0.05$ and $\mid$ foldchange $\mid>1.5$. Furthermore, to identify differentially expressed protein complex, we used Wilcoxon rank-sum test on the average protein expression profiles of protein complex members. Protein complexes with pvalue $\leq 0.05$ and $\mid$ foldchange $\mid>1$ were selected differential protein complex analysis.

\section{Pathway enrichment analysis}

The differentially expressed set of genes or proteins were tested for enrichment against gene sets of KEGG pathways present in Molecular Signature Database (MSigDB) v6.0 [39]. A hypergeometric test based over-representation analysis was used for this purpose (https://github.com/raunakms/GSEAFisher). A cut-off threshold of false discovery rate (FDR) $\leq 0.01$ was used to obtain the significantly enriched pathways. Only pathways that are enriched with at least four differentially expressed genes were considered for further analysis. To calculate the pathway activity score, the expression dataset was transformed into standard normal distribution using "inverse normal transformation" method. This step is necessary for a fair comparison between the expression-values of different genes. For each sample, the pathway activity score is the mean expression level of the differentially expressed genes linked to the enriched pathway. The KEGG signaling pathways and associated expression profile were visualized using pathview R-package [40].

\section{External datasets}

We utilized DNA sequencing datasets of publicly available patient LGSOC cohort from the American Association for Cancer Research (AACR) project Genomics Evidence Neoplasia Information Exchange (GENIE) [41]. The dataset consisted of a total of 122 LGSOC tumors (both primary and metastatic tumors). We used somatic mutation and copy number aberration profiles from the dataset. AACR project GENIE Data: Version 5.0 was downloaded from (SynapselD: syn7222066) https://www.synapse.org/\#!Synapse:syn7222066.

\section{Protein complex co-expression score}


For a given protein complex, its co-expression score is computed as the average Pearson correlation coefficient of all pairwise protein-protein interactions (as represented in STRING ver 10 database [38]) in the complex. The co-expression scores were separately calculated using mRNA expression data $\left(R_{m R N A}\right)$ and protein expression data $\left(R_{\text {protein }}\right)$. Protein complexes with $\left(\left|R_{\text {mrna }}-R_{\text {protien }}\right| \leq 0.05\right)$ are defined as correlated protein complexes. For this analysis, we considered the comprehensive resource of mammalian protein complexes (CORUM) protein complexes [42] consisting of at least 4 proteins.

\section{Results}

\section{Landscape of somatic mutations in LGSOC}

To investigate the landscape of somatic mutation changes in LGSOC cell lines, we performed highcoverage whole exome sequencing (WES) of 14 LGSOC cell lines and 10 matched normal samples from 11 independent patients ( 3 patients had 2 consecutive tumors cultured and included in this study) (Supplementary Table 1). We refer to this cohort of LGSOC cell lines as VGH cohort. We achieved a mean coverage of $106 x$ for normal samples and 107x for tumor samples (Supplementary Table 2). Altogether, we detected 1893 highly confident non-silent mutations in the protein-coding regions in 14 cell lines (Supplementary Table 3-4).

As described in our recent study [24], non-synonymous mutations in KRAS were found in all four MEKisensitive LGSOC cell lines (CL-01, CL-02, CL-14, and CL-15) (Fig. 1A). Two of them (CL-01 and CL-14) harbored $K R A S^{G 12 D}$ mutations while the other two (CL-02 and CL-15) harbored KRAS ${ }^{G 12 V}$ mutations (Fig. 1B-C). The variant allele frequency (VAF) of these KRAS mutations were over $70 \%$ in CL-14, CL-15, and CL-01 and that in CL-02 was 55\% (Supplementary Figure 1) indicating that these mutations are highly likely to be clonal in origin. Interestingly, all mutations detected in KRAS were the glycine residue at position 12. NRAS was mutated in one MEKi-sensitive cell line and three MEKi-resistant cell lines. $N R A S^{Q 61 R}$ mutation was specific to the three MEKi-resistant cell lines (CL-03, CL-04, CL-10) whereas, NRAS ${ }^{C 118 Y}$ mutation was present in one MEKi-sensitive cell line (CL-14) with a co-occurring KRAS mutation. The VAF of NRAS mutations ranged between $48-62 \%$ also indicating that these mutations are highly likely to be clonal. (Supplementary Figure 1). A $B R A F^{D 594 G}$ mutation was present in only one cell line (CL-09) (Fig. 1B). We further evaluated somatic mutations in a large cohort of LGSOC tumors ( $n=97$, includes both primary and metastatic tumors, but the tumor stages information are not available) in AACR project GENIE [41]. All mutations identified in KRAS, NRAS, and BRAF in the VGH cohort were localized in the same amino-acid positions as that reported in the GENIE cohort (Fig. 1C). However, BRAF ${ }^{D 594 G}$ 
mutation identified in the VGH cohort was not present in tumors GENIE cohort but one tumor sample harbored a mutation in the same amino acid position (BRAF ${ }^{D 594 N}$ ) (Supplementary Figure 2). Ten cell lines, including all MEKi-sensitive cases, had at least one mutated gene in the MAPK pathway (Supplementary Figure 3A). In the GENIE cohort, RAS/RAF mutations were prevalent (63\%) and were mutually exclusive (Supplementary Figure 2).

Apart from mutations in MAPK signaling pathway axis, other sparsely mutated genes identified in the LGSOC cell lines were involved in Notch pathway, chromatin remodeling, and DNA repair (Fig. 1A, Supplementary Figure 3A). Notably, TP53 ${ }^{\mathrm{R} 234 \mathrm{H}}$ mutations were observed in two MEKi-resistant cell lines (CL-07 and CL-08) both with VAF above 96\% indicating their clonal origin (Fig 1A, Supplementary Figure 1). While mutations in TP53 are rare in LGSOC tumors (8\% frequency) $[9,43]$, these cell lines (which were derived from two sequential tumor samples corresponding to the same patient) had been confirmed to be LGSOC by pathology review [44]. Furthermore, 3\% of tumors in the GENIE cohort had TP53 mutations. Notch pathway genes were mutated in 5 cell lines; mostly in MEKi-resistant cases. Signaling pathways such as PI3K, chromatin remodeling, MYC, TP53, and NRF2 were mostly mutated in MEKi-resistant cell lines (Supplementary Figure 3A). Similarly, different subsets of LGSOC tumors in GENIE cohort also harbored mutations in DNA repair pathway, chromatin remodeling pathway, Notch pathway, and TP53 pathway (Supplementary Figure 3B).

Using the software deconstructSigs [35], we evaluated the characteristic mutation patterns in our patientderived cell lines against the mutational signatures obtained from the COSMIC mutational signature database [36]. Analyses of the mutation patterns in the cell lines revealed strong enrichment of C>T transition nucleotide substitution mutations in MEKi-sensitive cell lines whereas MEKi-resistant and MEKi-untested cell lines were enriched in both $\mathrm{C}>\mathrm{T}$ (transition) as well as $\mathrm{C}>\mathrm{A}$ (transversion) nucleotide substitution mutations (Fig. 1A and Supplementary Figure 4). Moreover, GENIE cohort of LGSOC tumors also demonstrated strong enrichment of $\mathrm{C}>\mathrm{T}$ and $\mathrm{C}>\mathrm{A}$ nucleotide substitution mutations. We found mutational signature 1 , predominantly related to age-related mutagenesis, to be operative in MEKisensitive cell lines (Fig. 1A). On the other hand, mutational signatures 4, 8, 24, and 29 that are associated with transcriptional strand bias for $\mathrm{C}>\mathrm{A}$ mutations were operative in MEKi-resistant and MEKi-untested cell lines. Interestingly, mutational signatures 3, 6, and 15 that are associated with DNA mismatch repair were also operative in five MEKi-resistant and MEKi-untested cell lines. Clinical annotation of these samples revealed that MEKi-sensitive cell lines were associated with primary tumors (rather than recurrent tumors) that had received a lower number of therapy regimens prior collection than the MEKiresistant associated cultures. Interestingly, MEKi-sensitive cell lines, despite being associated with older 
patients (average 65.5 vs. 51.7 years), their estimated overall survival rates were found to be longer (average 6.5 vs. 4.4 years) (Supplementary Table 1). The presence of serous borderline or micropapillary tumor patterns, as well as the time to recurrence were not found to be associated with the two distinct MEKi response phenotype observed in our LGSOC cell lines.

Finally, using the data available from the GENIE cohort, we compared the exonic tumor mutation burden (TMB) in the four major subtypes of ovarian cancer (HGSOC, LGSOC, clear cell ovarian cancer, and endometrioid ovarian cancer) in the GENIE cohort. Compared to the other subtypes, LGSOC tumors have a low TMB (Fig. 1D).

\section{Landscape of copy number aberrations in LGSOC}

Copy number aberrations (CNAs) were determined using the whole exome sequence reads. We identified 6480 CNAs across 14 LGSOC cell lines analyzed (Supplementary Table 5). Copy number changes for all cell lines are shown in Fig. 2A, and those for individual cell lines in Fig. 2B. As previously described, loss of chromosome 9p and gains in chromosomes 8, 12 and 20 are frequent [22]. Overall, homozygous copy loss of MTAP was observed in 13 out of 14 LGSOC cell lines and its loss of heterozygosity (LOH) was observed in 1 cell line (Fig. 2C). Similarly, homozygous copy loss of CDKN2A/B was observed in 12 out of 14 LGSOC cell lines and its $\mathrm{LOH}$ was observed in 2 cell lines. These copy number changes were associated with loss of $\mathrm{p} 16$ expression by western blot (data available upon request).

Overall GISTIC analysis of focal amplifications and deletion revealed several recurrent events containing known oncogenic drivers (Supplementary Table 6). These include amplifications of PDGFRB (5q32), FGFR4 (5q35), MYC (8q24), CCND2 (12p13) along with deletions of CDKN2A (9p21), MAPK1 (22q11), and SMARCB1 (22q11). All four MEKi-sensitive LGSOC cell lines, which were KRAS mutant, also harbored copy-number gains affecting KRAS. CNA were identified in different genes targeting key oncogenic pathways such as MAPK, NOTCH, PI3K, Hippo, Wnt, TGF-beta signaling pathways, chromatin remodeling, DNA repair pathways, and cell cycle process (Supplementary Figure 5). We observed copy number deletions of few genes in Chromatin Remodeling, NOTCH, and Wnt signaling pathways enriched in MEKi-resistant cell lines. Interestingly, MEKi-sensitive cell lines are for the most part copy neutral on chromosome 1,9p, and 10 and also tend to have chromosome 5, 8, and 12 copy number gains. 


\section{Multiomics approaches to identify LGSOC drivers and predict MEKi-response}

\section{Identification of driver genes in LGSOC using HIT'nDRIVE}

Using our recently developed computational algorithm HIT'nDRIVE [37], we identified driver genes in these LGSOC cell lines. We first ran HIT'nDRIVE using SNV-mRNA expression data and CNA-mRNA expression data. HIT'nDRIVE analysis prioritized 17 unique driver genes in 8 LGSOC cell lines for which matched genome and transcriptome data were available (Fig. 3A and Supplementary Table 7). Similarly, we also ran HIT'nDRIVE using SNV-protein expression data and CNA-protein expression data. This analysis identified 19 unique driver genes in 7 LGSOC cell lines for which matched genome and proteome data were available (Fig. 3B and Supplementary Table 7).

Both HIT'nDRIVE analyses using mRNA and protein expression data identified genes in chromosome 9p21, MTAP, CDKN2A, and CDKN2B, as the most common driver genes among all LGSOC cell lines (Fig. 3A-B). For both CDKN2A and MTAP, the mRNA and protein expression profiles (available for 7 cell lines) were found to correlate and to be expressed at very low levels. Analysis of CNA in the LGSOC tumors in the GENIE cohort revealed that loss of CDKN2A/B and MTAP was present in about 31.5\% (17 out of 54 ) of all tumors, with few homozygous deletions $7.4 \%$ (4 out of 54) (Supplementary Table 8).

Using HIT'nDRIVE CNA-mRNA expression analysis copy number gains that were shared by more than one cell line include $A B C C 5, X R N 1, B R D T$, and NFE2. Similarly, HIT'nDRIVE CNA-protein expression analysis revealed CNA gain of DIDO1, TPD52, DDX51, and SMUG1 in more than one cell line. Interestingly, NEF2, DDX51, and SMUG1 are located on chromosome 12q indicating potential role of this region in LGSOC progression. Furthermore, HIT'nDRIVE CNA-protein expression analysis prioritized mutation and loss of gene EIF4G3 specific to MEKi-resistant phenotype.

\section{Evaluating gene and protein expression to predict MEKi-response}

Next, we performed total RNA-seq (Illumina NextSeq500) on 8 LGSOC cell lines with known sensitivity to MEKi treatment [22]. We detected 14.3 million reads on an average and over 27700 unique expressed genes across all samples. Principal components analysis (PCA) using the RNA-seq profiles obtained from each sample revealed that, except for the sample CL-01, all other MEKi-sensitive samples were clustered together whereas MEKi-resistance samples had high variance and thus were spread along the principal component axes (Fig. 4A). This suggests that the MEKi-sensitive samples are transcriptionally very similar to each other, whereas the MEKi-resistant samples are very diverse. 
Next, we performed proteome analysis by mass spectrometry on 7 LGSOC cell lines in triplicate and identified 5110 unique proteins. The PCA analysis on the mass spectrometry protein expression data also revealed two distinct clusters for MEKi-sensitive and MEKi-resistant samples (Fig. 4B). This demonstrates that the protein expression profiles of LGSOC cell lines discriminates the MEKi-sensitive and MEKi-resistant phenotype better than their mRNA expression profiles. Similar to the PCA in mRNA expression data, the CL-01 sample in PCA in the protein expression data was quite distinct as compared to the rest of the MEKi-sensitive samples.

We then identified differentially expressed transcripts and proteins between MEKi-sensitive and MEKiresistant phenotype (see Methods section). A total of 1,231 and 1,202 differentially expressed genes (in mRNA expression profiles) and proteins (in protein expression profiles) were respectively identified, out of which 113 gene/protein pairs were detected in both datasets (Supplementary Figure 6A and Supplementary Table 9-10). Similar to the results obtained from the PCA analysis, the expression profiles of the differentially expressed proteins discriminates the MEKi-sensitive and MEKi-resistant phenotype better than that of the differentially expressed genes (mRNA) (Supplementary Figure 6B-C). The differentially expressed genes/proteins between MEKi-sensitive and MEKi-resistant LGSOC cell lines included EGFR, PRKCA, MAPK4, NF2, MTOR, MTAP, ATM, TGFB2, and BRCA2.

To identify signaling pathways dysregulated by the differentially expressed genes/proteins between MEKi-sensitive and MEKi-resistant cell lines, we performed gene set enrichment analysis (see Methods section for details) (Supplementary Table 11-12). Although, distinct sets of genes (in the mRNA expression profile) and proteins (in the protein expression profile) were found to be differentially expressed between the MEKi-response phenotypes, intriguingly, we observed similar sets of differentially expressed signaling pathways enriched between the MEKi-response phenotypes between the two datasets (Fig. 4C-D). We found that majority of the proteins in the signaling pathways such as MAPK pathway, Wnt pathway, P53 pathway, DNA Replication, DNA Repair pathway, Cell cycle process, and Amino-acid metabolism were found to be highly expressed in MEKi-resistant as compared to MEKisensitive cell lines. On the other hand, we found majority of the proteins in Oxidative Phosphorylation and Tight Junction pathways to be highly expressed in the MEKi-sensitive lines. Similar to the observation made in Fig. 4A-B (and Supplementary Fig. 7), the pathway level analysis also indicates that the protein expression profiles separate the MEKi response phenotypes much more distinctly than that from mRNA expression data. Thus, in the downstream analysis we mostly present analysis based on protein expression profiles. 


\section{Protein complexes characterizing MEKi response phenotypes.}

As a novel discovery approach, we sought to identify large protein complexes characterizing the differences in the MEKi response phenotypes, we leveraged a curated set of core protein complexes from the CORUM database [42]. To identify protein complexes that characterize the MEKi response phenotypes, we performed Wilcoxon rank-sum test on the average protein expression profiles of CORUM protein complex members (see Methods section). In MEKi-resistant cell lines, we identified protein complexes such as BRCC complex, p130-ER-alpha complex, VEGFR2 complex, Condensin complex, minichromosome maintenance (MCM) complex and replication factor $C$ (RFC) complex to have high protein-expression of their complex members in MEKi-resistant cell lines (Fig. 5A-B and Supplemental Table 13). In MEKi-sensitive cell lines, we found F1F0-ATP synthase complex and RNA-induced silencing complex (RISC) to have high protein-expression of their complex members.

Furthermore, to identify protein complexes whose both transcript and protein of its members were coexpressed, we measured their co-expression score (see Methods section). We found 56 (or of 343) protein complexes with correlated mRNA and protein co-expression (Figure. 5C and Supplementary Table 14). We also found 28 protein complexes with high mRNA co-expression score $\left(R_{m R N A}>0.5\right)$ as well as high protein co-expression score $\left(R_{\text {protein }}>0.5\right)$. This included the Condensin complex which was found to have strong co-expression scores. MCM and RFC complexes on the other had strong protein co-expression but weak mRNA co-expression.

\section{Dysregulation of MAPK signaling pathway that may influence MEKi response}

With the knowledge that MEKi treatment shows activity in some patients with incurable LGSOC disease, we further investigated MAPK pathway activation in more detail. First, we correlated mRNA and protein expression fold change of genes/proteins involved in this pathway (Fig. 6A). In MEKi-resistant cell lines, genes such as PRKCA, HSPA2, TGFB2, FGF3, TP53, and FLNC were highly upregulated in MEKiresistant samples in both $\mathrm{mRNA}$ and protein expression data, and that these findings were highly correlated (Fig. 6B). On the other hand, MAPK13, HSPB1, and CACNA2D1 were upregulated in MEKisensitive cell lines. Furthermore, we found that the RAS-RAF-MEK-ERK axis was highly upregulated in the MEKi-resistant cell lines as compared to the MEKi-sensitive cell lines. (Supplementary Figure 8). 


\section{Discussion}

Fatality rates in women with LGSOC are high due to a lack of effective treatments. Our study is the first to use a multiomics approach to characterize of the genome, transcriptome [45-47], and proteome [4852] profiles of 14 patient-derived cell lines. These cell line models closely recapitulate the genomic abnormalities depicted in LGSOC tumors from the GENIE cohort [24] and other previous studies [5-7], and highlight common somatic mutations known to involve the MAPK (RAS/RAF/MEK) pathway $[10,41,53]$. LGSOC cell lines were characterized by a strong enrichment of $C>T$ and $C>A$ nucleotide substitution mutations, a predominance of KRAS mutations, and CNA's that are characteristic of those described in previous reports of LGSOC [10,11]. Both LGSOC cell lines and tumors were characterized by a lower tumor mutation burden than other ovarian cancer subtypes. Our findings also support earlier observations that BRAF mutations are less frequent than RAS mutations in advanced/recurrent LGSOC cases $[10,54,55]$.

Mutational signatures associated with LGSOC have not been previously described. Though these signatures have provided a remarkable basis for characterizing patterns of genomic disruption resulting from etiological factors in cancer generally [56], they may also be of importance as predictive biomarkers. However, more work needs to be done to identify if mutational signature might also have important implications for tumor classification and treatment management. Both LGSOC tumors and cell lines were found to be associated with transcriptional bias for $\mathrm{C}>\mathrm{A}$ transversion nucleotide substitution mutations in LGSOC, indicating guanine damage that is being repaired by transcription-coupled nucleotide excision repair. Interestingly mutational signatures also distinguished MEKi response phenotypes in LGSOC cell lines. In particular, mutational signatures 1, 5, and 12 were associated with MEKi-sensitive cell lines, while signatures 8, 4, and 29 were much more prevalent in MEKi-resistant cell lines. Mutational signature 1 , which is associated with age, correlated with older patients.

Loss of the chromosome 9p21 tumor suppressor locus (including CDKN2A and MTAP) is the most common deletion event across many human cancer types [57-61]. Both LGSOC cell lines and tumors had deletions affecting these genes. However, we noted an important difference in the frequency of these events. As previously described, LGSOC cell lines (86\%; 12/14) had focal/homozygous deletions in chromosome 9p21, confirmed by a low CDKN2A/p16 expression. In contrast, LGSOC tumors from the GENIE project harbored heterozygous deletions of 9p21 in only 31.5-53\% of cases. Thus, there appears to be a selective advantage for establishing primary cultures/cell lines of LGSOC with $9 p$ loss. 
Interestingly, absence of $\mathrm{p} 16$ expression had been previously associated with an unfavorable prognosis in LGSOC [10,62] suggesting a potential value for the use of cell cycle inhibitors (CDK4) in LGSOC.

MTAP is a key enzyme in the methionine salvage pathway and is frequently co-deleted with $C D K N 2 A$ due to its chromosomal proximity [63,64]. From large scale short hairpin RNA-mediated screens across multiple human cancer cell lines, it is known that viability of MTAP-deficient cancer cells is impaired by depletion of the protein arginine methyltransferase PRMT5 [58,63]. Inhibitors of PRMT5 may be synthetically lethal strategy for this dysregulated metabolic state and are of interest in MTAP and $C D K N 2 A / B$-deleted tumors. The potential therapeutic value of MTAP and CDKN2A deficiency in LGSOC remains to be explored.

Our study describes several different techniques to evaluate multiomics data for the identification of disease drivers and MEKi-response prediction. Recently, Schutte et al. showed integration of genomic and transcriptomic data significantly outperformed less comprehensive analysis in the identification of clinically relevant molecular alterations [65]. The combination of DNA and RNA-based analyses have shown to serve as mutual controls for verifying potential findings [21]. As LGSOC is a rare cancer, model systems and patient samples are limited. Thus, efficient analytical tools and large datasets are needed to reduce false discovery rates and improve prediction accuracy. HIT'nDRIVE analysis exploits the power of analyzing multiomics data and can also be used to enhance discovery in rare cancers [66]. Using HIT'nDRIVE analysis we identified novel potential driver genes (KRAS, MTAP, and CDKN2A/B) in LGSOC cell lines. In recent clinical trial with LGSOC patients, KRAS mutation were found to be associated with better response to MEK inhibition with significant improvement in PFS with binimetinib when compared to physician choice of chemotherapy [67]. Other single case reports have shown similar findings $[68,69]$. While promising results of MEKi therapy for the treatment of LGSOC patients have been demonstrated in recent studies [70,71], biomarkers that predict treatment efficacy are largely unknown.

Here using LGSOC cell lines, we demonstrated that proteomic profiles were better at discriminating distinct MEKi-response phenotypes than transcriptomic profiles, and found that protein alterations affecting TP53, cell cycle and DNA-repair pathways are common in MEKi-resistant cell lines. These pathways should be further studied for their potential therapeutic value. Proteomic technologies are useful for drug prediction [72]. The addition of proteomic-based analysis facilitates the understanding of the biological implications of genomic change and can be used as discovery tools for both drug prediction and identification of novel therapeutic targets. We found considerable consistency in the identification of differential signaling pathway activity between MEKi-response phenotypes using proteomic and 
transcriptomic data, strongly suggesting that these pathways play a role in MEKi resistance. Recent developments in analytical approaches using machine learning also show promise in improving multiomics drug prediction [73].

MEKi will soon be commonly prescribed, for the treatment of LGSOC, as an alternative to the standard chemotherapy and anti-hormone therapy in patients with recurrent disease [74]. To identify robust biomarkers that predict MEKi response, we used transcript/protein expression to evaluate MAPK pathway regulation. MAPK signaling feedback loops play a key role in MEKi resistance [75]. Both PI3K-AKTmTOR pathway (Supplementary Figure 9) and MAPK pathway activity are upregulated in MEKiresistant cell lines. The most differentially expressed protein identified in the MAPK pathway analysis was protein kinase $\mathrm{C}$ alpha (PRKCA or PKC-a). We previously identified and validated both PKC-a and EGFR as biomarkers of MEKi resistance using reverse phase protein array (RPPA) [24]. This observation supports the utility of multiomics analysis approaches for predictive biomarker discovery as we used a completely different proteomic technologies and dataset for discovery. This and other novel predictive biomarkers of MEKi efficacy have been described and some of these biomarkers could be considered for validation in future clinical trials.

While MEKi-sensitive LGSOC lines depend on MAPK pathway activation for survival (as shown by the lethal effects of MEKi treatment in-vitro), the importance of MAPK in MEKi-resistant lines remains unclear. In the absence of protein phosphorylation data, we cannot assess the extent of MAPK signaling activation in the MEKi-resistant cell lines. However, a more comprehensive evaluation of the function (activator/inhibitor) and activation status (phosphorylated/not phosphorylated) of the protein candidates identified in here will allow researchers to clarify if MEKi-resistant lines will benefit of drug combinations targeting different proteins within the MAPK pathway (e.g. MEK plus BRAF inhibitors), or if alternatively, they will require the use of other targeted therapies to achieve tumor cell death.

Finally, we used our proteomic data identified protein complexes with potential therapeutic relevance in LGSOC. Using the CORUM database, we elucidated novel protein complexes differentially expressed between the MEKi response phenotypes. Previous studies have shown that some of these protein complexes regulate cell proliferation; including RFC in ovarian cancer [76] and MCM in other cancers $[77,78]$. The MCM complex forms the core of the DNA replicative helicase and, interestingly, is associated with cisplatin resistance [79]. Increased RFC expression has been shown to be associated with epithelialmesenchymal transition and poor outcomes in both lung and breast cancer [80]. Other complexes we identified, such as the BRCC and the Condesin complexes, have been linked to DNA repair in cancer 
[81,82]. Although, MEKi represents a promising new therapy in LGSOC, MEKi treatment is only associated with a 6-8 months improvement in PFS. Increased expression of proteins within the complexes found in MEKi resistant cell lines provides a strong rationale to further explore these complexes as potential therapeutic targets.

LGSOC is a rare and often lethal cancer for which research model development has been challenging. As a result, our study is limited in the number of samples available for analysis. Data is not currently available to validate our findings in patients treated with MEKi. In this context, we have used multiomics analysis approaches as a disease driver and drug prediction biomarker discovery tool. Our study cohort has a higher frequency of $\mathrm{p} 16$ loss (chromosome 9p loss) than what is described in LGSOC tumors and thus models that preserve p16 expression will be needed for evaluation.

\section{Conclusion}

Using a multiomics approach, we have characterized the largest worldwide collection of LGSOC cell lines to improve their use as research model systems. These cell lines accurately reflect the molecular makeup of LGSOC tumor samples characterized to date though LGSOC cell lines without 9p loss are not adequately represented in our sample. We have also described key molecular aberrations in our cell lines (including signaling pathway identification) that describe potential biomarkers of MEKi response and novel potential disease drivers. We found that proteomic data was more robust in differentiating MEKi drug sensitivity and identified several protein complexes that warrant further study in LGSOC. This approach has the potential to accelerate the discovery of clinically useful biomarkers and novel drug targets in MEKi-treated patient populations. Multiomics analytical approaches show great promise to improve our current understanding of cancer biology, drug therapy, and ultimately patient management. 


\section{Additional files}

Additional file 1: Supplementary material. (PDF 1.6 MB)

Additional file 2: Supplementary tables. (XLSX 1.3 MB)

\section{Abbreviations}

AACR: American Association for Cancer Research; AF: Allele frequencies; BAM: Binary alignment map; CNA: Copy number aberration; CORUM: Comprehensive resource of mammalian protein complexes; ER: Estrogen receptor; FDR: False discovery rate; GENIE: Genomics Evidence Neoplasia Information Exchange; HGSOC: High-grade serous ovarian carcinoma; HPLC: High performance liquid chromatography; LGSOC: Low-grade serous ovarian carcinoma; LOH: Loss of heterozygosity; MCM: Minichromosome maintenance; MEKi: MEK inhibitors; MS: Mass spectrometry; MSigDB: Molecular signature database; OC: Ovarian Cancer; PCR: Polymerase chain reaction; PECA: Probe-level expression change averaging; PFS: Progression free survival; PKC-a: protein kinase C alpha; PR: Progesterone receptor; RFC: Replication factor C-alpha; RISC: RNA-induced silencing complex SNV: Single nucleotide variation; TMB: Tumor mutation burden; TMT: Tandem mass tag; UBC: University of British Columbia; VAF: Variant allele frequency; VGH: Vancouver General Hospital; WES: Whole exome sequencing

\section{Acknowledgements}

The authors thanks all members of the Carey's and the Collin's labs for helpful suggestions. The results published here are in part based upon data generated by the AACR Project GENIE https://www.synapse.org/\#!Synapse:syn7222066.

\section{Funding}

This study is funded by the Canada Foundation for Innovation (CFI-IF 33440), Terry Fox Research Institute (TFRI NF PPG Project \#1062) (C.C.C), British Columbia Cancer Foundation (M.S.C) and the OvCaRe research program. The authors want to extend a special thanks to the MacKenzie and Lawler families, the Janet D. Cottrelle Foundation, and to all patients, families, and donors who supported this research. We would also like to thank the Society of Gynecologic Oncology of Canada (GOC) for their support in advancing research and knowledge translation in LGSOC, the Canadian Institutes of Health 
Research (CHIR) for their Planning and Disseminating grant support (NRF 152680), and the Women's Health Research Institute (WHRI) for their Catalyst Grant support.

\section{Availability of data and materials}

The whole exome and whole transcriptome sequencing data from this study is available in the European Genome-phenome Archive (EGA) under accession number XXXXXXX. The proteome data from mass spectrometry proteome data is available in the PRIDE Archive under accession number PXD019544.

\section{Authors' contributions}

RS, MLF, CCC, and MSC conceived the study and wrote the manuscript. RS, JH, SV, YL SA, BM, and $\mathrm{RHB}$ performed data analysis. ML, AD, HK, AH, SC, BC, SB, GED, and GBM performed the experiments generating the cell line models, specimen processing, quality control, sequencing, and mass spectrometry experiments. SLB, GBM, CCC, and MSC supervised the project, contributed scientific insights, and edited the manuscript. All authors read, contributed, and approved the final manuscript.

\section{Ethics approval and consent to participate}

This study was approved by institutional human ethics review boards at BCCA (H14-02859), the University of British Columbia (UBC; R05-0119), and the University of Western Ontario (HSREB 12668E).

\section{Consent for publication}

Not applicable.

\section{Competing interests}

The authors declare that they have no competing interests. 


\section{References}

1. Gershenson DM. Low-grade serous carcinoma of the ovary or peritoneum. Ann Oncol Off J Eur Soc Med Oncol [Internet]. 2016;27 Suppl 1:i45-9. Available from: http://www.ncbi.nlm.nih.gov/pubmed/27141071

2. Grabowski JP, Harter P, Heitz F, Pujade-Lauraine E, Reuss A, Kristensen G, et al. Operability and chemotherapy responsiveness in advanced low-grade serous ovarian cancer. An analysis of the AGO Study Group metadatabase. Gynecol Oncol [Internet]. 2016;140:457-62. Available from: http://www.ncbi.nlm.nih.gov/pubmed/26807488

3. Shih I-M, Kurman RJ. Ovarian tumorigenesis: a proposed model based on morphological and molecular genetic analysis. Am J Pathol [Internet]. American Society for Investigative Pathology; 2004;164:1511-8. Available from: http://dx.doi.org/10.1016/S0002-9440(10)63708-X

4. Köbel M, Kalloger SE, Boyd N, McKinney S, Mehl E, Palmer C, et al. Ovarian carcinoma subtypes are different diseases: implications for biomarker studies. PLoS Med [Internet]. 2008;5:e232. Available from: http://www.ncbi.nlm.nih.gov/pubmed/19053170

5. Singer G, Oldt R, Cohen Y, Wang BG, Sidransky D, Kurman RJ, et al. Mutations in BRAF and KRAS characterize the development of low-grade ovarian serous carcinoma. J Natl Cancer Inst [Internet]. 2003;95:484-6. Available from: http://www.ncbi.nlm.nih.gov/pubmed/10822068

6. Wong K-K, Tsang YTM, Deavers MT, Mok SC, Zu Z, Sun C, et al. BRAF mutation is rare in advancedstage low-grade ovarian serous carcinomas. Am J Pathol [Internet]. American Society for Investigative Pathology; 2010;177:1611-7. Available from: http://dx.doi.org/10.2353/ajpath.2010.100212

7. Emmanuel C, Chiew Y-E, George J, Etemadmoghadam D, Anglesio MS, Sharma R, et al. Genomic classification of serous ovarian cancer with adjacent borderline differentiates RAS pathway and TP53mutant tumors and identifies NRAS as an oncogenic driver. Clin Cancer Res [Internet]. 2014;20:661830. Available from: http://www.ncbi.nlm.nih.gov/pubmed/25316818

8. Singer G, Stöhr R, Cope L, Dehari R, Hartmann A, Cao D-F, et al. Patterns of p53 mutations separate ovarian serous borderline tumors and low- and high-grade carcinomas and provide support for a new model of ovarian carcinogenesis: a mutational analysis with immunohistochemical correlation. Am J Surg Pathol [Internet]. 2005;29:218-24. Available from: http://www.ncbi.nlm.nih.gov/pubmed/15644779

9. Van Nieuwenhuysen E, Busschaert P, Laenen A, Moerman P, Han SN, Neven P, et al. Loss of 1p36.33 Frequent in Low-Grade Serous Ovarian Cancer. Neoplasia [Internet]. 2019;21:582-90. Available from: http://www.ncbi.nlm.nih.gov/pubmed/31054497

10. Hunter SM, Anglesio MS, Ryland GL, Sharma R, Chiew Y-E, Rowley SM, et al. Molecular profiling of low grade serous ovarian tumours identifies novel candidate driver genes. Oncotarget [Internet]. 
2015;6:37663-77. Available from: http://www.ncbi.nlm.nih.gov/pubmed/26506417

11. Rambau PF, Vierkant RA, Intermaggio MP, Kelemen LE, Goodman MT, Herpel E, et al. Association of 16 expression with prognosis varies across ovarian carcinoma histotypes: an Ovarian Tumor Tissue Analysis consortium study. J Pathol Clin Res [Internet]. 2018;250-61. Available from: http://www.ncbi.nlm.nih.gov/pubmed/30062862

12. Buttarelli M, Mascilini F, Zannoni GF, Ciucci A, Martinelli E, Filippetti F, et al. Hormone receptor expression profile of low-grade serous ovarian cancers. Gynecol Oncol [Internet]. 2017;145:352-60. Available from: http://www.ncbi.nlm.nih.gov/pubmed/28228232

13. Escobar J, Klimowicz AC, Dean M, Chu P, Nation JG, Nelson GS, et al. Quantification of ER/PR expression in ovarian low-grade serous carcinoma. Gynecol Oncol [Internet]. 2013;128:371-6. Available from: http://www.ncbi.nlm.nih.gov/pubmed/23103384

14. Wong K-K, Lu KH, Malpica A, Bodurka DC, Shvartsman HS, Schmandt RE, et al. Significantly greater expression of ER, PR, and ECAD in advanced-stage low-grade ovarian serous carcinoma as revealed by immunohistochemical analysis. Int J Gynecol Pathol [Internet]. 2007;26:404-9. Available from: http://www.ncbi.nlm.nih.gov/pubmed/17885490

15. Etemadmoghadam D, Azar WJ, Lei Y, Moujaber T, Garsed DW, Kennedy CJ, et al. EIF1AX and NRAS Mutations Co-occur and Cooperate in Low-Grade Serous Ovarian Carcinomas. Cancer Res [Internet]. 2017;77:4268-78. Available from: http://www.ncbi.nlm.nih.gov/pubmed/28646021

16. Prat J. New insights into ovarian cancer pathology. Ann Oncol [Internet]. 2012;23:x111-7. Available from: https://linkinghub.elsevier.com/retrieve/pii/S0923753419417535

17. Gershenson DM, Sun CC, Bodurka D, Coleman RL, Lu KH, Sood AK, et al. Recurrent low-grade serous ovarian carcinoma is relatively chemoresistant. Gynecol Oncol [Internet]. Elsevier Inc.; 2009;114:48-52. Available from: http://dx.doi.org/10.1016/j.ygyno.2009.03.001

18. Gershenson DM, Sun CC, Iyer RB, Malpica AL, Kavanagh JJ, Bodurka DC, et al. Hormonal therapy for recurrent low-grade serous carcinoma of the ovary or peritoneum. Gynecol Oncol [Internet]. 2012;125:661-6. Available from: http://www.ncbi.nlm.nih.gov/pubmed/22406638

19. Marquart J, Chen EY, Prasad V. Estimation of the Percentage of US Patients With Cancer Who Benefit From Genome-Driven Oncology. JAMA Oncol [Internet]. 2018;4:1093-8. Available from: http://oncology.jamanetwork.com/article.aspx?doi=10.1001/jamaoncol.2018.1660

20. Massard C, Michiels S, Ferté C, Le Deley M-C, Lacroix L, Hollebecque A, et al. High-Throughput Genomics and Clinical Outcome in Hard-to-Treat Advanced Cancers: Results of the MOSCATO 01 Trial. Cancer Discov [Internet]. 2017;7:586-95. Available from: http://www.ncbi.nlm.nih.gov/pubmed/28365644 21. Rodon J, Soria J-C, Berger R, Miller WH, Rubin E, Kugel A, et al. Genomic and transcriptomic profiling expands precision cancer medicine: the WINTHER trial. Nat Med [Internet]. 2019;25:751-8. Available 
from: http://www.nature.com/articles/s41591-019-0424-4

22. Fernández ML, DiMattia GE, Dawson A, Bamford S, Anderson S, Hennessy BT, et al. Differences in MEK inhibitor efficacy in molecularly characterized low-grade serous ovarian cancer cell lines. Am J Cancer Res [Internet]. 2016;6:2235-51. Available from: http://www.ncbi.nlm.nih.gov/pubmed/27822414 23. Mert I, Chhina J, Allo G, Dai J, Seward S, Carey MS, et al. Synergistic effect of MEK inhibitor and metformin combination in low grade serous ovarian cancer. Gynecol Oncol [Internet]. 2017;146:319-26. Available from: http://www.ncbi.nlm.nih.gov/pubmed/28545687

24. Fernandez ML, Dawson A, Hoenisch J, Kim H, Bamford S, Salamanca C, et al. Markers of MEK inhibitor resistance in low-grade serous ovarian cancer: EGFR is a potential therapeutic target. Cancer Cell Int [Internet]. BioMed Central; 2019;19:10. Available from: https://doi.org/10.1186/s12935-019-07251

25. Li H, Durbin R. Fast and accurate short read alignment with Burrows-Wheeler transform. Bioinformatics [Internet]. 2009;25:1754-60. Available from: http://www.ncbi.nlm.nih.gov/pubmed/19451168

26. Kim S, Scheffler K, Halpern AL, Bekritsky MA, Noh E, Källberg M, et al. Strelka2: fast and accurate calling of germline and somatic variants. Nat Methods [Internet]. Springer US; 2018;15:591-4. Available from: http://dx.doi.org/10.1038/s41592-018-0051-x

27. Wilm A, Aw PPK, Bertrand D, Yeo GHT, Ong SH, Wong CH, et al. LoFreq: a sequence-quality aware, ultra-sensitive variant caller for uncovering cell-population heterogeneity from high-throughput sequencing datasets. Nucleic Acids Res [Internet]. 2012;40:11189-201. Available from: http://www.ncbi.nlm.nih.gov/pubmed/23066108

28. Wang K, Li M, Hakonarson H. ANNOVAR: functional annotation of genetic variants from highthroughput sequencing data. Nucleic Acids Res [Internet]. 2010;38:e164. Available from: http://www.ncbi.nlm.nih.gov/pubmed/20601685

29. Dobin A, Davis C a., Schlesinger F, Drenkow J, Zaleski C, Jha S, et al. STAR: ultrafast universal RNA-seq aligner. Bioinformatics [Internet]. 2013;29:15-21. Available from: http://www.ncbi.nlm.nih.gov/pubmed/23104886

30. Anders S, Pyl PT, Huber W. HTSeq--a Python framework to work with high-throughput sequencing data. Bioinformatics [Internet]. 2015;31:166-9. Available from: http://www.ncbi.nlm.nih.gov/pubmed/25260700

31. Anders S, Huber W. Differential expression analysis for sequence count data. Genome Biol [Internet]. 2010;11:R106. Available from: http://genomebiology.biomedcentral.com/articles/10.1186/gb-2010-1110-r106

32. UniProt Consortium T. UniProt: the universal protein knowledgebase. Nucleic Acids Res [Internet]. 
2018;46:2699. Available from: http://www.ncbi.nlm.nih.gov/pubmed/29425356

33. Leek JT, Johnson WE, Parker HS, Jaffe AE, Storey JD. The sva package for removing batch effects and other unwanted variation in high-throughput experiments. Bioinformatics [Internet]. 2012;28:882-3. Available from: http://www.ncbi.nlm.nih.gov/pubmed/22257669

34. Teo G, Vogel C, Ghosh D, Kim S, Choi H. PECA: a novel statistical tool for deconvoluting timedependent gene expression regulation. J Proteome Res [Internet]. 2014;13:29-37. Available from: http://www.ncbi.nlm.nih.gov/pubmed/24229407

35. Rosenthal R, McGranahan N, Herrero J, Taylor BS, Swanton C. DeconstructSigs: delineating mutational processes in single tumors distinguishes DNA repair deficiencies and patterns of carcinoma evolution. Genome Biol [Internet]. Genome Biology; 2016;17:31. Available from: http://genomebiology.com/2016/17/1/31

36. Alexandrov LB, Jones PH, Wedge DC, Sale JE, Campbell PJ, Nik-Zainal S, et al. Clock-like mutational processes in human somatic cells. Nat Genet [Internet]. Nature Publishing Group; 2015;47:1402-7. Available from: http://dx.doi.org/10.1038/ng.3441

37. Shrestha R, Hodzic E, Sauerwald T, Dao P, Wang K, Yeung J, et al. HIT'nDRIVE: patient-specific multidriver gene prioritization for precision oncology. Genome Res [Internet]. 2017;27:1573-88. Available from: http://genome.cshlp.org/lookup/doi/10.1101/gr.221218.117

38. Szklarczyk D, Franceschini A, Wyder S, Forslund K, Heller D, Huerta-Cepas J, et al. STRING v10: protein-protein interaction networks, integrated over the tree of life. Nucleic Acids Res [Internet]. 2015;43:D447-52. Available from: http://nar.oxfordjournals.org/lookup/doi/10.1093/nar/gku1003

39. Subramanian A, Tamayo P, Mootha VK, Mukherjee S, Ebert BL, Gillette MA, et al. Gene set enrichment analysis: a knowledge-based approach for interpreting genome-wide expression profiles. Proc Natl Acad Sci U S A [Internet]. 2005 [cited 2014 Jul 14];102:15545-50. Available from: http://www.pubmedcentral.nih.gov/articlerender.fcgi?artid=1239896\&tool=pmcentrez\&rendertype=abstr act

40. Luo W, Brouwer C. Pathview: an R/Bioconductor package for pathway-based data integration and visualization. Bioinformatics [Internet]. 2013;29:1830-1. Available from: http://www.ncbi.nlm.nih.gov/pubmed/23740750

41. AACR Project GENIE Consortium. AACR Project GENIE: Powering Precision Medicine through an International Consortium. Cancer Discov [Internet]. 2017;7:818-31. Available from: http://www.ncbi.nlm.nih.gov/pubmed/28572459\%5Cnhttp://cancerdiscovery.aacrjournals.org/lookup/doi/ 10.1158/2159-8290.CD-17-0151

42. Giurgiu M, Reinhard J, Brauner B, Dunger-Kaltenbach I, Fobo G, Frishman G, et al. CORUM: the comprehensive resource of mammalian protein complexes-2019. Nucleic Acids Res [Internet]. 
2019;47:D559-63. Available from: http://www.ncbi.nlm.nih.gov/pubmed/19884131

43. Romero I, Sun CC, Wong KK, Bast RC, Gershenson DM. Low-grade serous carcinoma: new concepts and emerging therapies. Gynecol Oncol [Internet]. Elsevier Inc.; 2013;130:660-6. Available from: http://dx.doi.org/10.1016/j.ygyno.2013.05.021

44. Chay WY, Horlings HM, Tinker A V., Gelmon KA, Gilks CB. Low grade serious carcinoma of the peritoneum in a BRCA1 carrier previously diagnosed with a "low-grade serous tubal intra-epithelial carcinoma" (STIC) on risk reducing surgery. Gynecol Oncol reports [Internet]. Elsevier B.V.; 2015;12:724. Available from: http://dx.doi.org/10.1016/j.gore.2015.03.007

45. Quu C, Lu N, Wang X, Zhang Q, Yuan C, Yan S, et al. Gene expression profiles of ovarian low-grade serous carcinoma resemble those of fallopian tube epithelium. Gynecol Oncol [Internet]. 2017;147:63441. Available from: http://www.ncbi.nlm.nih.gov/pubmed/28965696

46. Wang C, Winterhoff BJ, Kalli KR, Block MS, Armasu SM, Larson MC, et al. Expression signature distinguishing two tumour transcriptome classes associated with progression-free survival among rare histological types of epithelial ovarian cancer. Br J Cancer [Internet]. 2016;114:1412-20. Available from: http://www.ncbi.nlm.nih.gov/pubmed/27253175

47. Curry EWJ, Stronach EA, Rama NR, Wang YYP, Gabra H, El-Bahrawy MA. Molecular subtypes of serous borderline ovarian tumor show distinct expression patterns of benign tumor and malignant tumorassociated signatures. Mod Pathol [Internet]. 2014;27:433-42. Available from: http://www.ncbi.nlm.nih.gov/pubmed/23948749

48. Zadeh Fakhar HB, Zali H, Rezaie-Tavirani M, Darkhaneh RF, Babaabasi B. Proteome profiling of low grade serous ovarian cancer. J Ovarian Res [Internet]. 2019;12:64. Available from: http://www.ncbi.nlm.nih.gov/pubmed/31315664

49. Garg G, Yilmaz A, Kumar P, Turkoglu O, Mutch DG, Powell MA, et al. Targeted metabolomic profiling of low and high grade serous epithelial ovarian cancer tissues: a pilot study. Metabolomics [Internet]. 2018;14:154. Available from: http://www.ncbi.nlm.nih.gov/pubmed/30830441

50. Sans M, Zhang J, Lin JQ, Feider CL, Giese N, Breen MT, et al. Performance of the MasSpec Pen for Rapid Diagnosis of Ovarian Cancer. Clin Chem [Internet]. 2019;65:674-83. Available from: http://www.ncbi.nlm.nih.gov/pubmed/30770374

51. Klein O, Kanter F, Kulbe H, Jank P, Denkert C, Nebrich G, et al. MALDI-Imaging for Classification of Epithelial Ovarian Cancer Histotypes from a Tissue Microarray Using Machine Learning Methods. Proteomics Clin Appl [Internet]. 2019;13:e1700181. Available from: http://www.ncbi.nlm.nih.gov/pubmed/30471200

52. Spreafico A, Oza AM, Clarke BA, Mackay HJ, Shaw P, Butler M, et al. Genotype-matched treatment for patients with advanced type I epithelial ovarian cancer (EOC). Gynecol Oncol [Internet]. 
2017;144:250-5. Available from: http://www.ncbi.nlm.nih.gov/pubmed/28062115

53. Tone AA, McConechy MK, Yang W, Ding J, Yip S, Kong E, et al. Intratumoral heterogeneity in a minority of ovarian low-grade serous carcinomas. BMC Cancer [Internet]. 2014;14:982. Available from: http://www.ncbi.nlm.nih.gov/pubmed/25523272

54. Tsang YT, Deavers MT, Sun CC, Kwan S-Y, Kuo E, Malpica A, et al. KRAS (but not BRAF) mutations in ovarian serous borderline tumour are associated with recurrent low-grade serous carcinoma. J Pathol [Internet]. 2013;231:449-56. Available from: http://www.ncbi.nlm.nih.gov/pubmed/24549645

55. Grisham RN, lyer G, Garg K, Delair D, Hyman DM, Zhou Q, et al. BRAF mutation is associated with early stage disease and improved outcome in patients with low-grade serous ovarian cancer. Cancer [Internet]. 2013;119:548-54. Available from: http://www.ncbi.nlm.nih.gov/pubmed/22930283

56. Alexandrov LB, Nik-Zainal S, Wedge DC, Aparicio S a. JR, Behjati S, Biankin A V., et al. Signatures of mutational processes in human cancer. Nature [Internet]. 2013 [cited 2013 Aug 14];500:415-21. Available from: http://www.nature.com/doifinder/10.1038/nature12477

57. LaPak KM, Burd CE. The molecular balancing act of p16(INK4a) in cancer and aging. Mol Cancer Res [Internet]. 2014;12:167-83. Available from: http://www.ncbi.nlm.nih.gov/pubmed/24136988

58. Mavrakis KJ, McDonald ER, Schlabach MR, Billy E, Hoffman GR, DeWeck A, et al. Disordered methionine metabolism in MTAP/CDKN2A-deleted cancers leads to dependence on PRMT5. Science [Internet]. 2016;351:1208-13. Available from: http://www.ncbi.nlm.nih.gov/pubmed/26912361

59. Fedoriw A, Rajapurkar SR, O’Brien S, Gerhart S V, Mitchell LH, Adams ND, et al. Anti-tumor Activity of the Type I PRMT Inhibitor, GSK3368715, Synergizes with PRMT5 Inhibition through MTAP Loss. Cancer Cell [Internet]. 2019;36:100-114.e25. Available from: http://www.ncbi.nlm.nih.gov/pubmed/31257072

60. Kohno T, Yokota J. Molecular processes of chromosome 9p21 deletions causing inactivation of the p16 tumor suppressor gene in human cancer: deduction from structural analysis of breakpoints for deletions. DNA Repair (Amst) [Internet]. 2006;5:1273-81. Available from: http://www.ncbi.nlm.nih.gov/pubmed/16931177

61. Collins CC, Volik S V, Lapuk A V, Wang Y, Gout PW, Wu C, et al. Next generation sequencing of prostate cancer from a patient identifies a deficiency of methylthioadenosine phosphorylase, an exploitable tumor target. Mol Cancer Ther [Internet]. 2012;11:775-83. Available from: http://www.ncbi.nlm.nih.gov/pubmed/22252602

62. Konecny GE, Winterhoff B, Kolarova T, Qi J, Manivong K, Dering J, et al. Expression of p16 and retinoblastoma determines response to CDK4/6 inhibition in ovarian cancer. Clin Cancer Res [Internet]. 2011;17:1591-602. Available from: http://www.ncbi.nlm.nih.gov/pubmed/21278246

63. Kryukov G V, Wilson FH, Ruth JR, Paulk J, Tsherniak A, Marlow SE, et al. MTAP deletion confers 
enhanced dependency on the PRMT5 arginine methyltransferase in cancer cells. Science [Internet]. 2016;351:1214-8. Available from: http://www.ncbi.nlm.nih.gov/pubmed/26912360

64. Marjon K, Cameron MJ, Quang P, Clasquin MF, Mandley E, Kunii K, et al. MTAP Deletions in Cancer Create Vulnerability to Targeting of the MAT2A/PRMT5/RIOK1 Axis. Cell Rep [Internet]. The Authors; 2016;15:574-87. Available from: http://dx.doi.org/10.1016/j.celrep.2016.03.043

65. Schütte M, Risch T, Abdavi-Azar N, Boehnke K, Schumacher D, Keil M, et al. Molecular dissection of colorectal cancer in pre-clinical models identifies biomarkers predicting sensitivity to EGFR inhibitors. Nat Commun [Internet]. 2017;8:14262. Available from: http://www.ncbi.nlm.nih.gov/pubmed/28186126 66. Shrestha R, Nabavi N, Lin Y-Y, Mo F, Anderson S, Volik S, et al. BAP1 haploinsufficiency predicts a distinct immunogenic class of malignant peritoneal mesothelioma. Genome Med [Internet]. Genome Medicine; 2019;11:8. Available from: http://dx.doi.org/10.1186/s13073-019-0620-3

67. Grisham RN, Moore KN, Gordon MS, Harb W, Cody G, Halpenny DF, et al. Phase Ib study of binimetinib with paclitaxel in patients with platinum-resistant ovarian cancer: Final results, potential biomarkers, and extreme responders. Clin Cancer Res. 2018;24:5525-33.

68. Takekuma M, Wong KK, Coleman RL. A long-term surviving patient with recurrent low-grade serous ovarian carcinoma treated with the MEK1/2 inhibitor, selumetinib. Gynecol Oncol Res Pract [Internet]. Gynecologic Oncology Research and Practice; 2016;3:1-5. Available from: http://dx.doi.org/10.1186/s40661-016-0026-5

69. Han C, Bellone S, Zammataro L, Schwartz PE, Santin AD. Binimetinib (MEK162) in recurrent lowgrade serous ovarian cancer resistant to chemotherapy and hormonal treatment. Gynecol Oncol reports [Internet]. 2018;25:41-4. Available from: http://www.ncbi.nlm.nih.gov/pubmed/29946554

70. Farley J, Brady WE, Vathipadiekal V, Lankes HA, Coleman R, Morgan MA, et al. Selumetinib in women with recurrent low-grade serous carcinoma of the ovary or peritoneum: An open-label, Singlearm, Phase 2 study. Lancet Oncol [Internet]. Elsevier Ltd; 2013;14:134-40. Available from: http://dx.doi.org/10.1016/S1470-2045(12)70572-7

71. Arend RC, Davis AM, Chimiczewski P, O’Malley DM, Provencher D, Vergote I, et al. EMR 20006012: A phase II randomized double-blind placebo controlled trial comparing the combination of pimasertib (MEK inhibitor) with SAR245409 (PI3K inhibitor) to pimasertib alone in patients with previously treated unresectable borderline or low grade. Gynecol Oncol [Internet]. Elsevier Inc.; 2020;156:301-7. Available from: https://doi.org/10.1016/j.ygyno.2019.12.002

72. Ali M, Khan SA, Wennerberg K, Aittokallio T. Global proteomics profiling improves drug sensitivity prediction: results from a multi-omics, pan-cancer modeling approach. Bioinformatics [Internet]. 2018;34:1353-62. Available from: http://www.ncbi.nlm.nih.gov/pubmed/29186355

73. Sharifi-Noghabi H, Zolotareva O, Collins CC, Ester M. MOLI: multi-omics late integration with deep 
neural networks for drug response prediction. Bioinformatics [Internet]. 2019;35:i501-9. Available from: http://www.ncbi.nlm.nih.gov/pubmed/31510700

74. Pauly N, Ehmann S, Ricciardi E, Ataseven B, Bommert M, Heitz F, et al. Low-grade Serous Tumors: Are We Making Progress? Curr Oncol Rep [Internet]. 2020;22:8. Available from: http://www.ncbi.nlm.nih.gov/pubmed/31989304

75. Lake D, Corrêa SAL, Müller J. Negative feedback regulation of the ERK1/2 MAPK pathway. Cell Mol Life Sci [Internet]. 2016;73:4397-413. Available from: http://www.ncbi.nlm.nih.gov/pubmed/27342992 76. Shen H, Xu J, Zhao S, Shi H, Yao S, Jiang N. ShRNA-mediated silencing of the RFC3 gene suppress ovarian tumor cells proliferation. Int J Clin Exp Pathol [Internet]. 2015;8:8968-75. Available from: http://www.ncbi.nlm.nih.gov/pubmed/26464638

77. Lei M. The MCM complex: its role in DNA replication and implications for cancer therapy. Curr Cancer Drug Targets [Internet]. 2005;5:365-80. Available from: http://www.ncbi.nlm.nih.gov/pubmed/16101384 78. Drissi R, Chauvin A, McKenna A, Lévesque D, Blais-Brochu S, Jean D, et al. Destabilization of the MiniChromosome Maintenance (MCM) complex modulates the cellular response to DNA double strand breaks. Cell Cycle [Internet]. 2018;17:2593-609. Available from: http://www.ncbi.nlm.nih.gov/pubmed/30516086

79. Deng M, Sun J, Xie S, Zhen H, Wang Y, Zhong A, et al. Inhibition of MCM2 enhances the sensitivity of ovarian cancer cell to carboplatin. Mol Med Rep [Internet]. 2019;20:2258-66. Available from: http://www.ncbi.nlm.nih.gov/pubmed/31322224

80. He Z-Y, Wu S-G, Peng F, Zhang Q, Luo Y, Chen M, et al. Up-Regulation of RFC3 Promotes Triple Negative Breast Cancer Metastasis and is Associated With Poor Prognosis Via EMT. Transl Oncol [Internet]. 2017;10:1-9. Available from: http://www.ncbi.nIm.nih.gov/pubmed/27888707

81. Dong Y, Hakimi MA, Chen X, Kumaraswamy E, Cooch NS, Godwin AK, et al. Regulation of BRCC, a holoenzyme complex containing BRCA1 and BRCA2, by a signalosome-like subunit and its role in DNA repair. Mol Cell. 2003;12:1087-99.

82. Kim JH, Youn Y, Kim KT, Jang G, Hwang JH. Non-SMC condensin I complex subunit H mediates mature chromosome condensation and DNA damage in pancreatic cancer cells. Sci Rep [Internet]. Springer US; 2019;9:1-12. Available from: http://dx.doi.org/10.1038/s41598-019-54478-3 


\section{Figures}

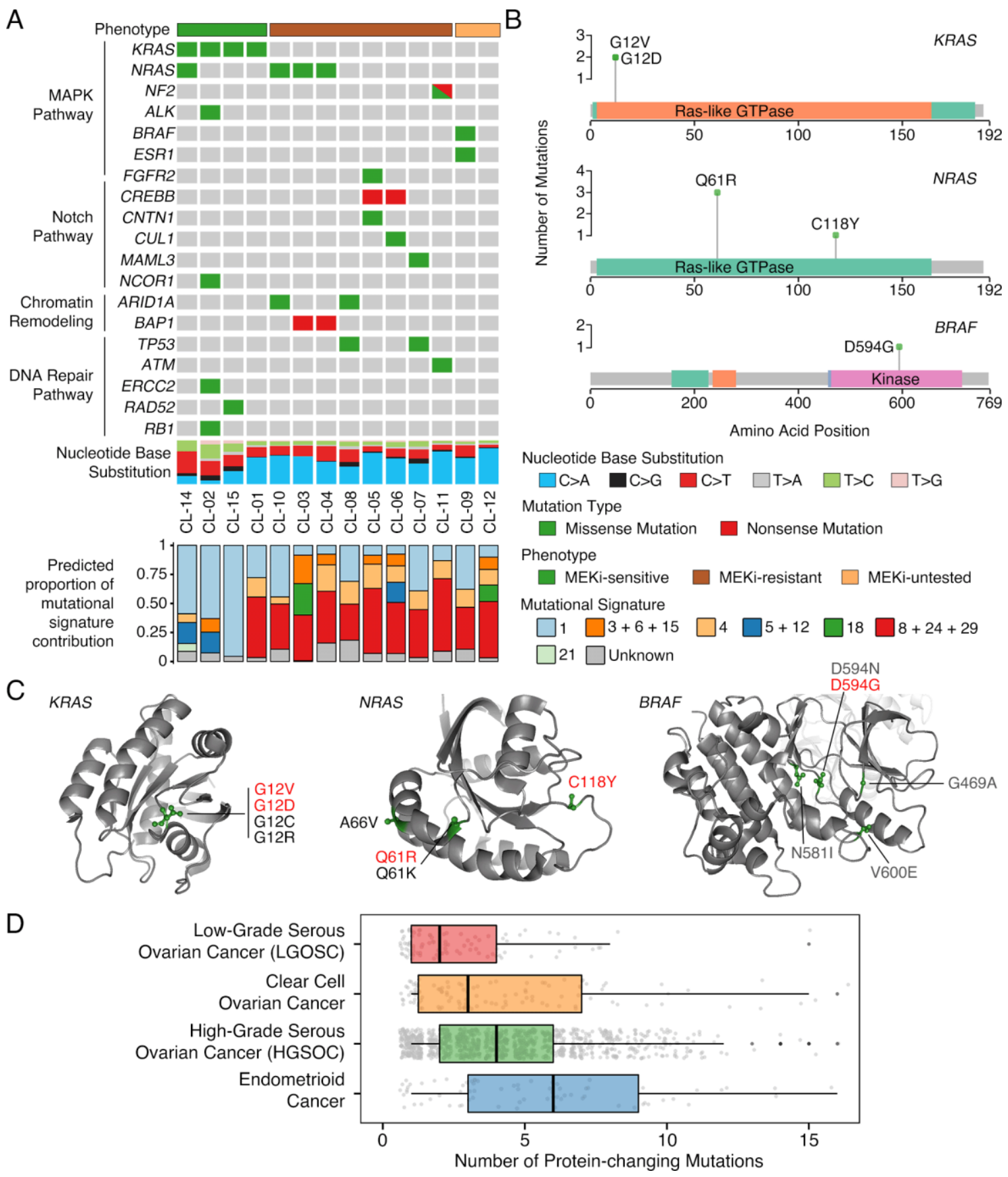

Figure 1. Landscape of somatic mutation in LGSOC. (A) Status of somatic mutations in fourteen LGSOC cell lines grouped by genes in major cancer pathways - MAPK pathway, Notch pathway, chromatin remodeling, and DNA repair pathway. The bottom panel represents the proportional contribution of different COSMIC mutational signatures per sample. (B) Plots showing mutation distribution and the protein domains for the corresponding mutated protein. (C) 3D protein structure of KRAS, NRAS, and BRAF with mutations identified in LGSOC cases in both VGH cohort (LGSOC cell lines, highlighted in red) and GENIE cohorts (LGSOC tumors) were highlighted. (D) Box plot showing the comparison of the tumor mutation burden in LGSOC with that of major subtypes of ovarian cancer. We leveraged the tumors included in the GENIE cohort for this analysis. Each dot in the plot represents a tumor sample of respective ovarian cancer subtype. 


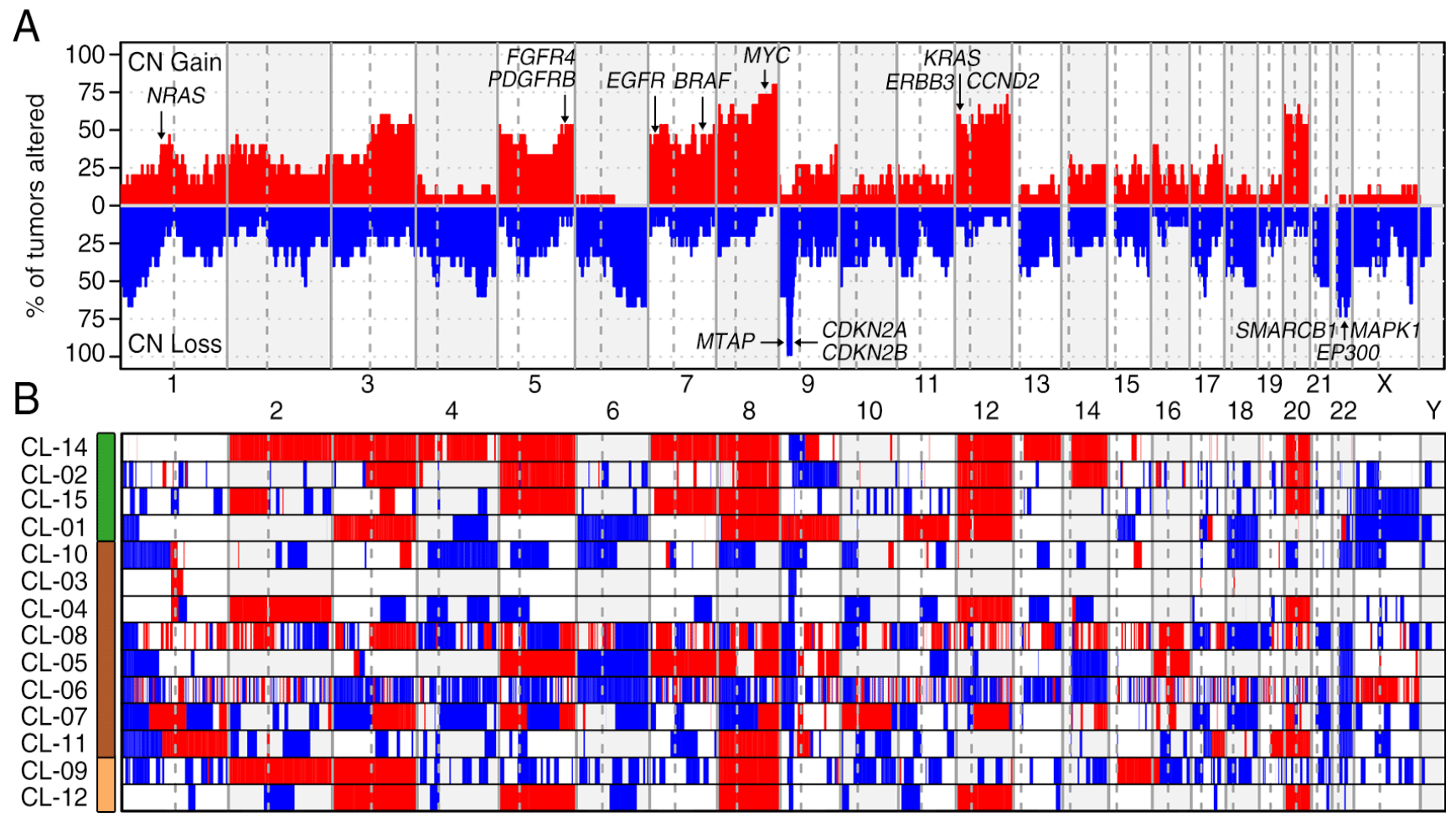

C

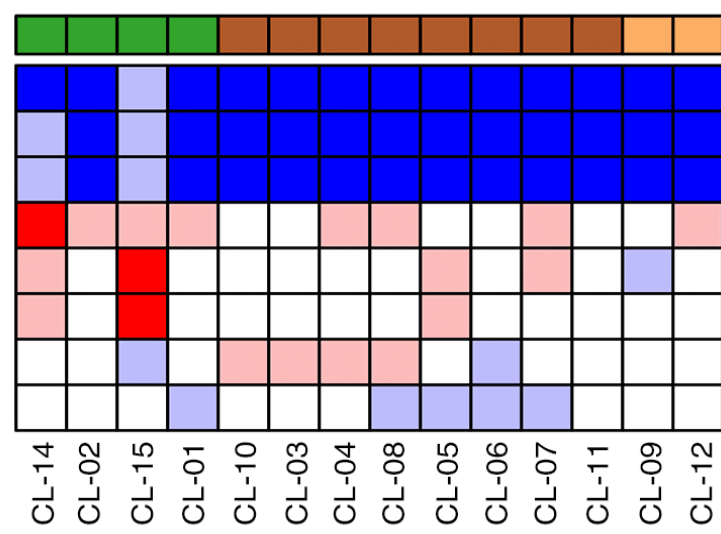

Phenotype

MTAP CDKN2A $\quad$ Chr 9p21 CDKN2B KRAS EGFR BRAF NRAS ESR1
MAPK pathway
Phenotype

$\square$ MEKi-sensitive

$\square$ MEKi-resistant

MEKi-untested

Copy Number Aberration

High copy number gain Copy number gain Copy number neutral Copy number loss Homozygous copy loss

Figure 2. Landscape of copy number aberrations in LGSOC. (A) Aggregate copy number alteration profile of LGSOC cell lines analyzed shown by each chromosome region. Red indicates copy number gain and blue indicates copy number loss. Important genes with copy number changes are highlighted. (B) Copy number alteration profile per sample analyzed. (C) Copy number status of some selected genes in chromosome 9p21 and MAPK pathway. 
A

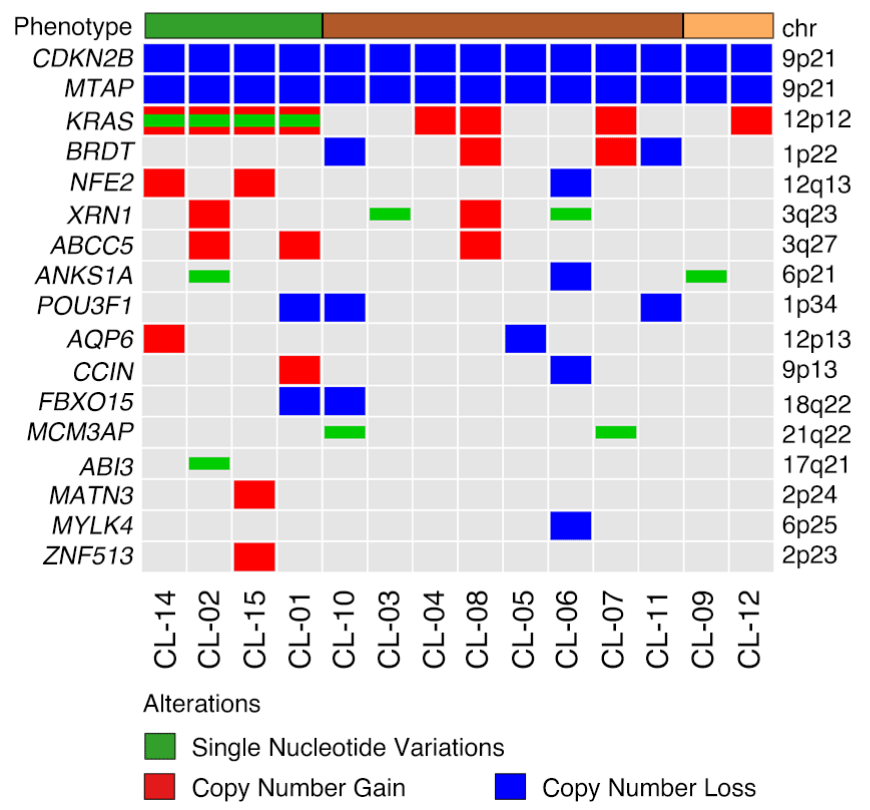

$\mathrm{B}$

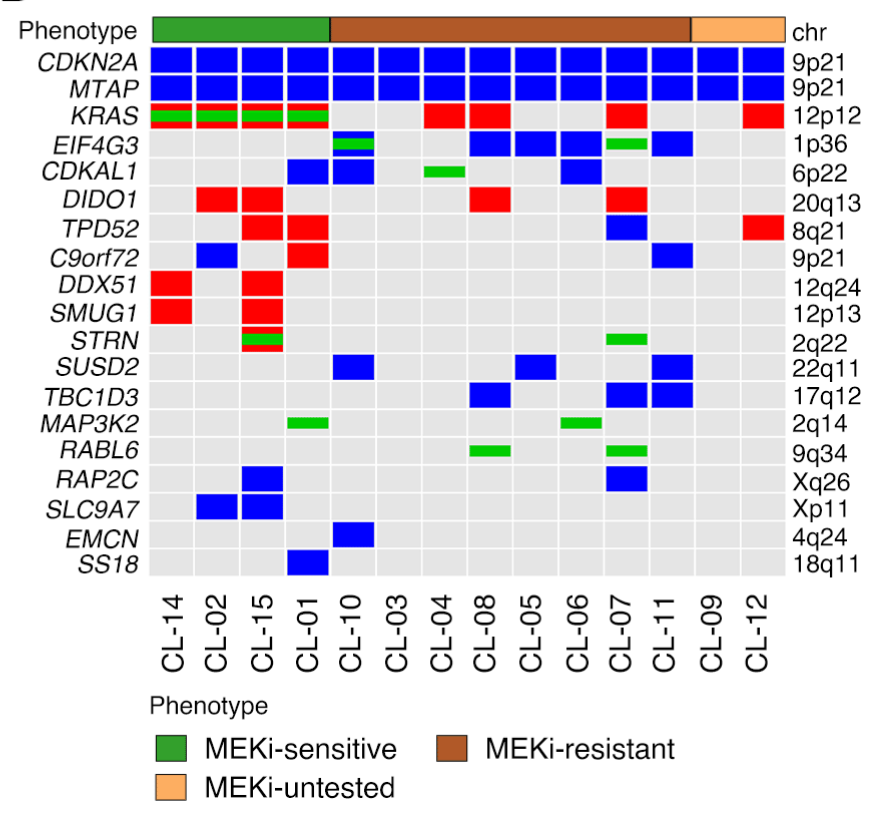

Figure 3. Driver genes of LGSOC identified by HIT'nDRIVE. (A-B) These oncoplots represent the genomic alterations in the driver genes of LGSOC identified by HIT'nDRIVE. To identify the driver genes, SNV and CNA data were used along with (A) mRNA expression data, and (B) protein expression data. Mutation and copy number aberration data were analyzed separately using HIT'nDRIVE. We note that: HIT'nDRIVE analysis were performed in (A) 8 LGSOC cell lines with matched genome and mRNA expression data and (B) 7 LGSOC cell lines with matched genome and protein expression data available. i.e. we could not use 6 cell lines without mRNA-expression profiles and 7 cell lines without protein-expression profile for HIT'nDRIVE analysis. However, in these cell lines not used for HIT'nDRIVE analysis, if the predicted driver genes resulting from other cell lines were present, we represented them as a part of the above figure. 
bioRxiv preprint doi: https://doi.org/10.1101/2020.06.18.135061; this version posted June 20, 2020. The copyright holder for this preprint (which was not certified by peer review) is the author/funder. All rights reserved. No reuse allowed without permission.

A

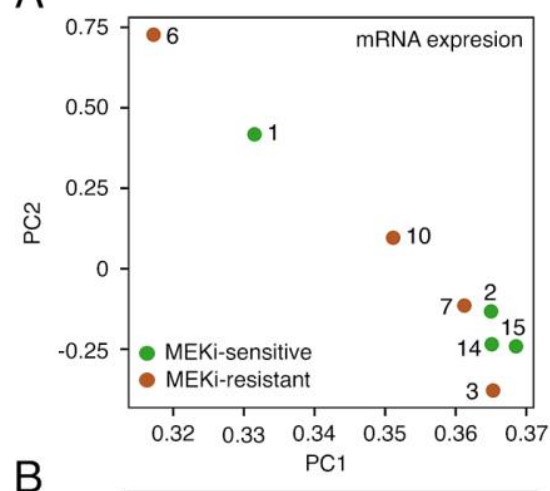

B

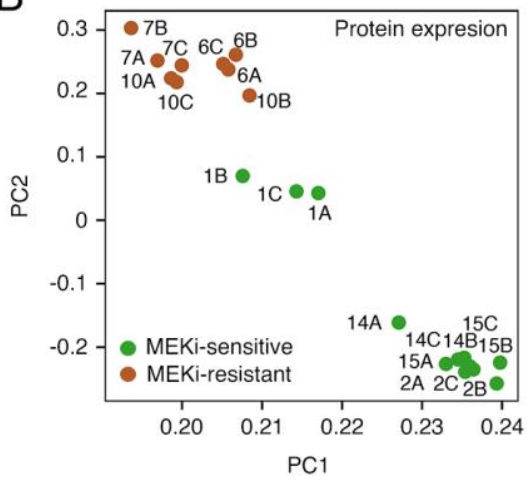

C
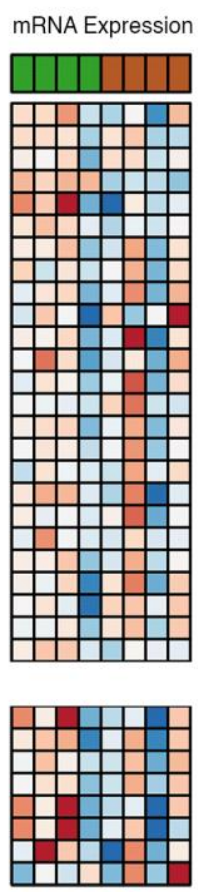

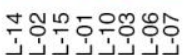

Pathway Activity Score

MEKi-sensitive

MEKi-resistant

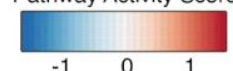

Oxidative phosphorylation

Alanine aspartate and glutamate metabolism Tyrosine metabolism

Peroxisome

Glyoxylate metabolism

Glycerophospholipid metabolism

Tight junction

Phosphatidylinositol signaling system

Pyruvate metabolism

Histidine metabolism

Adherens junction

Arginine and Proline metabolism

Pathways in Cancer

MAPK signaling

WNT signaling

Focal adhesion

PI3K-AKT signaling pathway

T-cell receptor signaling

B-cell receptor signaling

P53 signaling

TGFB signaling

ERBB signaling

Ubiquitin mediated proteolysis

Purine metabolism

Pyrimidine metabolism

Base excision repair

DNA replication

Nucleotide excision repair

Mismatch repair

Spliceosome

Cell cycle

Homologous recombination

Regulation of autophagy

Terpenoid backbone biosynthesis

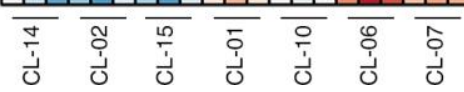
Pentose phosphate pathway

Figure 4. Characterization of transcriptome and proteome profile of MEKi response phenotypes. (A-B) Principal component analysis (PCA) of LGSOC using (A) 4710 most variable genes in mRNA expression profiles and (B) 1278 most variable proteins in proteome expression profiles. See Methods section for details. (C) Pathways enrichment of differentially expressed genes/proteins between LGSOC MEKi treatment phenotypes obtained using mRNA expression and protein expression (biological triplicates). 
A

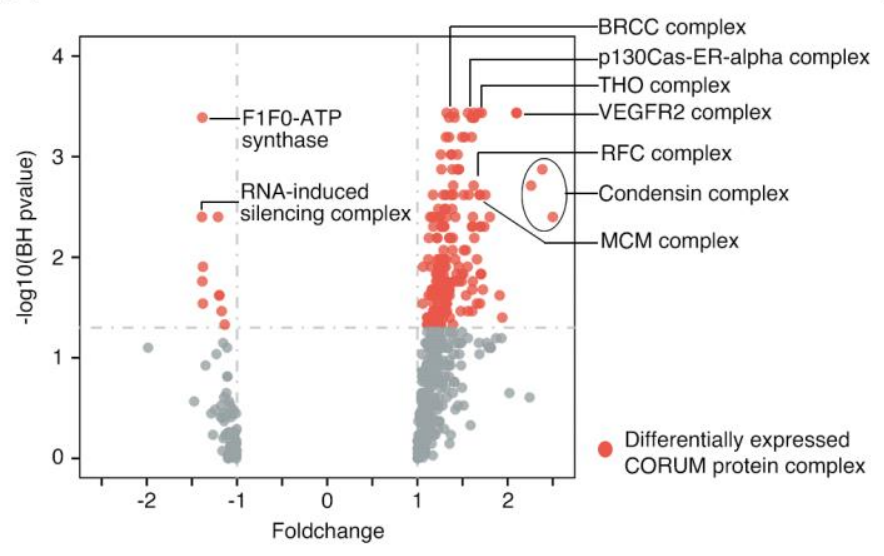

B

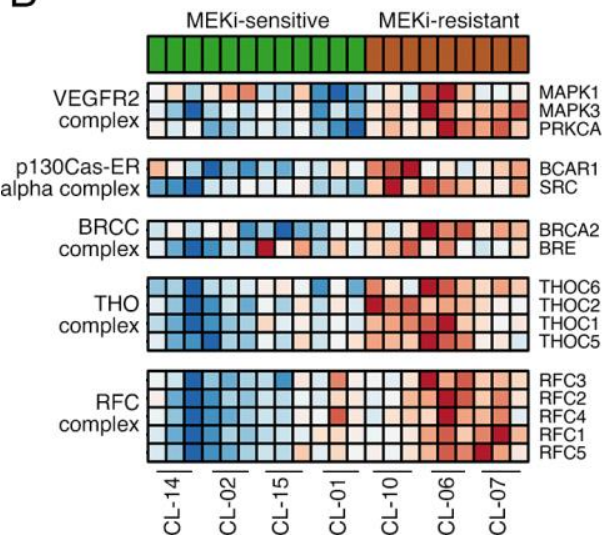

C

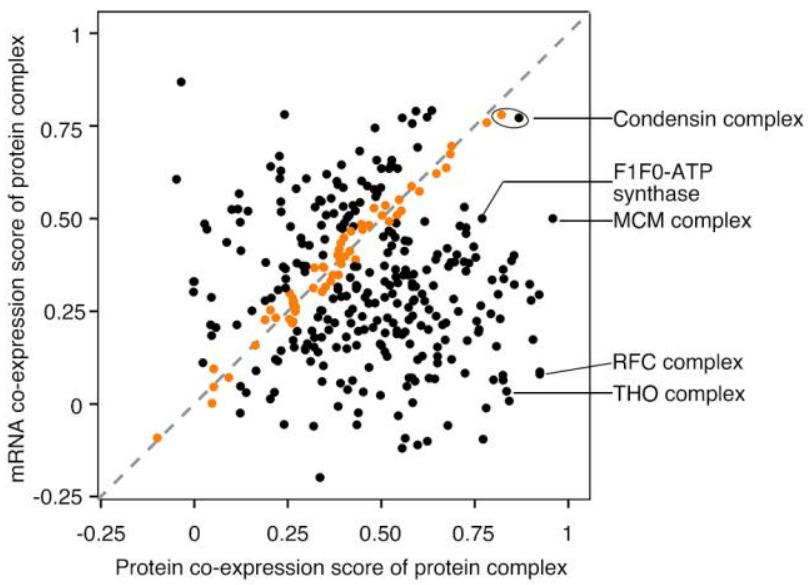

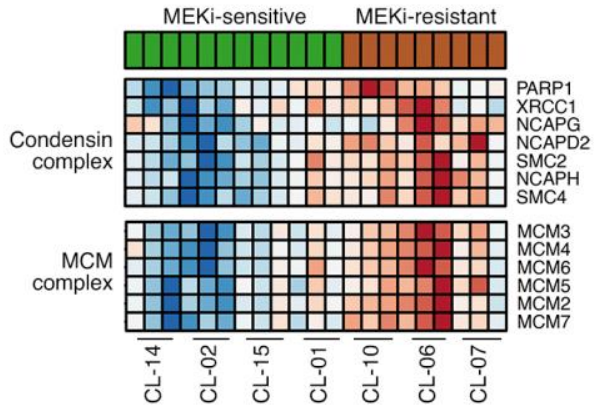

protein expression
MEKi-sensitive MEKi-resistant

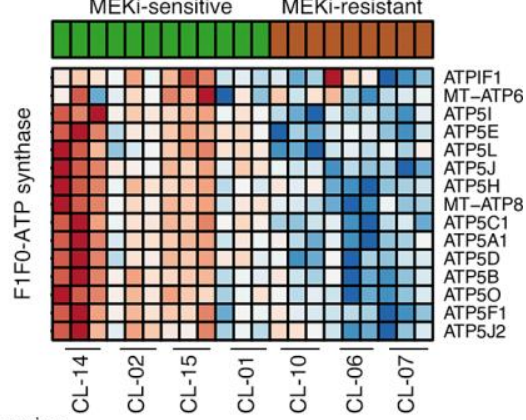

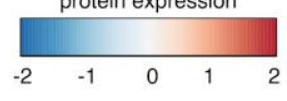

Figure 5. Protein complexes characterizing MEKi response phenotypes. (A) Volcano plot showing the differentially expressed CORUM protein complexes between MEKi sensitive and resistant LGSOC cell lines. Each dot represents a CORUM protein complex. Differentially expressed protein complexes are represented as red colored dots. The expression fold-change is calculated relative to MEKi-resistant cell lines. (B) Heatmap of protein expression profiles of CORUM protein complexes that are differentially expressed between the MEKi response phenotypes across LGSOC cell lines. (C) Scatter plot showing the correlation between mRNA co-expression score and protein co-expression score of CORUM protein complexes. Each dot in the figure represents a protein complex and the correlated protein complexes are highlighted in orange color. p130Cas-ER-alpha complex: p130Cas-ERalpha-cSrc-kinase- PI3-kinase p85-subunit complex; VEGFR2 complex: VEGFR2-S1PR1-ERK1/2-PKC-alpha complex. 
A

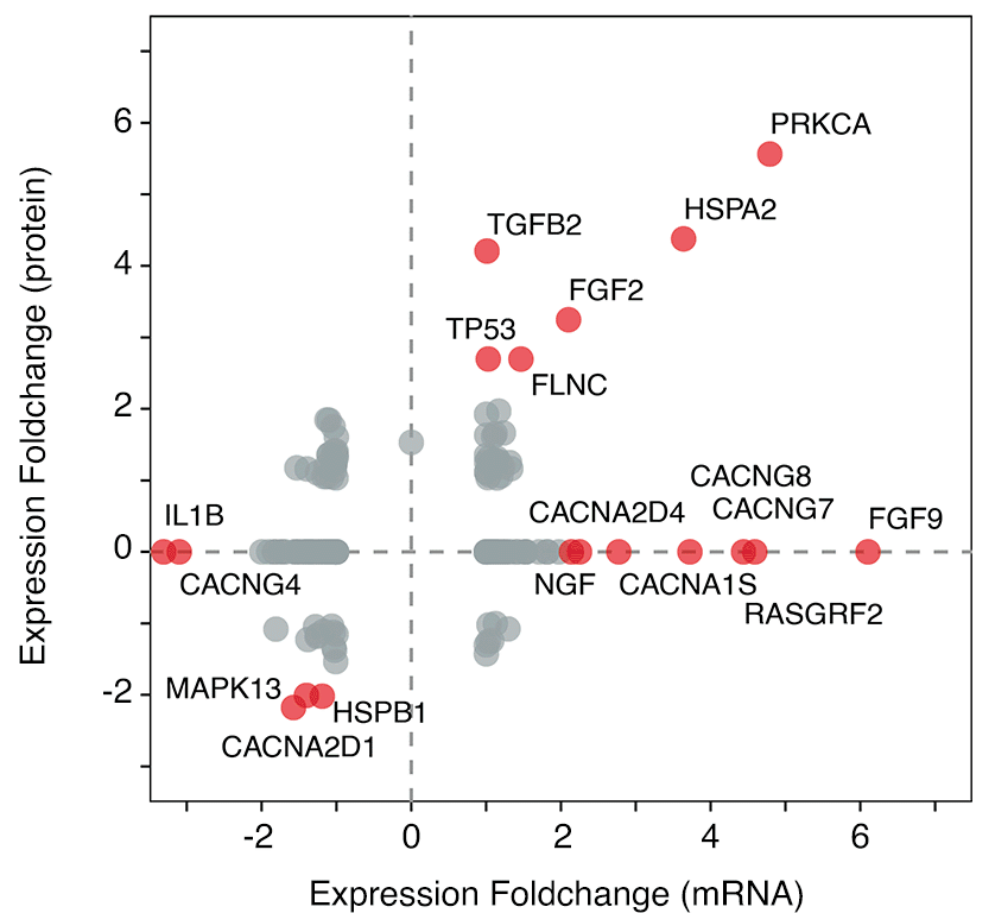

$\mathrm{B}$

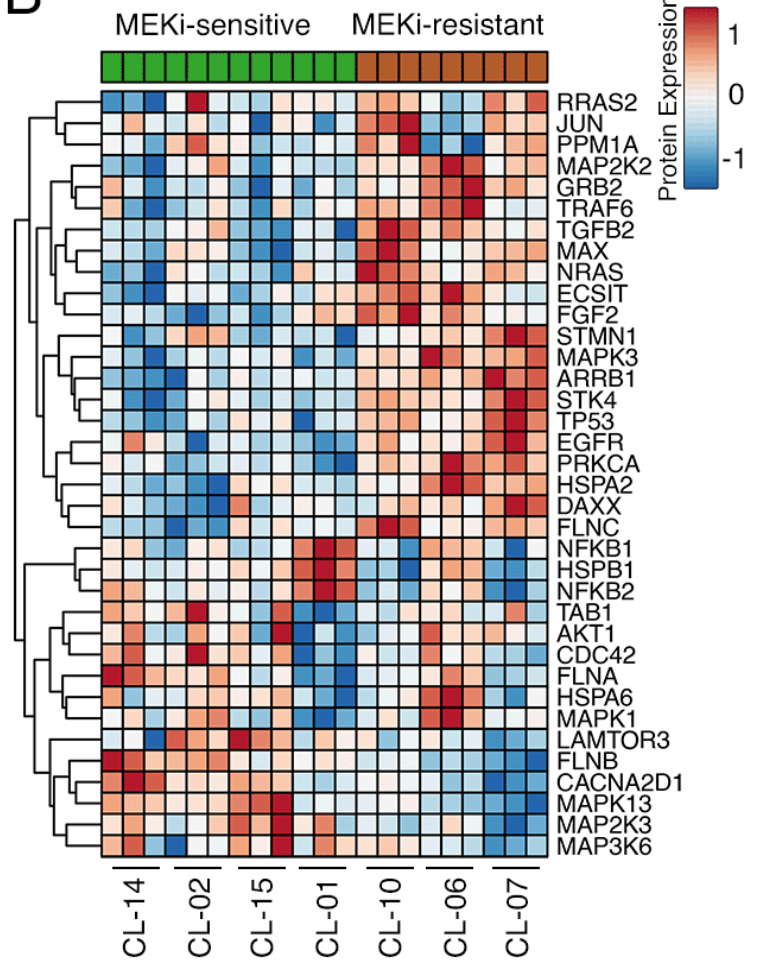

Figure 6. Proteome expression profile of MAPK signaling pathway characterizes response to MEKi treatment in LGSOC. (A) mRNA/Protein expression fold-change of genes or proteins involved in MAPK signaling pathway. Each dot represents a gene/protein. The expression fold-change is calculated relative to MEKi-resistant cell lines. The dots highlighted in red color are those genes/proteins with higher than 2 fold-change expression between the MEKi-resistant vs MEKi-sensitive LGSOC samples. (B) Heatmap of protein expression profiles of those proteins involved in MAPK signaling pathway. 